\title{
Cruciferae (Brassicaceae): Alternative treatment for the "Conspectus of the vascular plants of Mongolia" (2014)
}

\section{Cruciferae (Brassicaceae): альтернативная обработка для «Конспекта сосудистых растений Монголии» (2014)}

\author{
D.A. German \\ Д.А. Герман \\ Центр исследований организмов, Гейдельбергский университет, Им Нойенхаймер Фельд, 345; D-69120, Гейдельберг, \\ Германия; Южно-Сибирский ботанический сад, Алтайский государственный университет, пр-т Ленина, 61; 656049, \\ Барнаул, Россия. E-mail: oreoloma@rambler.ru \\ Centre for Organismal Studies, Heidelberg University, Im Neuenheimer Feld 345; D-69120 Heidelberg, Germany; \\ South-Siberian Botanical Garden, Altai State University, Lenina str., 61; 656049, Barnaul, Russia
}

Key words: Central Asia, phytodiversity, floristic findings, new combinations, systematics, Guenthera, Matthiola, Stevenia.

Ключевые слова: Центральная Азия, фиторазнообразие, флористические находки, новые комбинации, систематика, Guenthera, Matthiola, Stevenia.

Summary. The checklist of the mustards (Cruciferae, or Brassicaceae) of Mongolia supplied with the data on their distribution according to the phytogeographical regions of the country is presented which includes 141 species and 7 subspecies from 59 genera. Both taxonomic diversity and geography of Mongolian Cruciferae are critically revised with use of herbarium material, online resources, and published data. The genus Matthiola W. T. Aiton represented by the natively growing $M$. superba Conti is recorded from Mongolia for the first time and several regional floristic novelties are reported as well. On the other hand, newly and previously revealed erroneous state and regional records published mainly after 1996 are listed and commented. Finally, new combinations in the genera Guenthera Andrz. and Stevenia Fisch. are validated. The article can be regarded as an alternative treatment for the recent "Conspectus of the vascular plants of Mongolia" (2014).

Аннотация. Представлен конспект крестоцветных (Cruciferae, или Brassicaceae) Монголии, основанный на критической ревизии гербарных сборов, интернет-ресурсов и литературы, в том числе новейших работ по систематике семейства. Для всех 141 вида и 7 подвидов из 59 родов, отмеченных на данный момент во флоре страны, приведена краткая синонимика и данные по распространению (наличию или отсутствию в каждом из 16-ти ботанико-географических регионов) в Монголии. Впервые для страны указан род левкой - Matthiola W. T. Aiton, представленный естественно произрастающим здесь $M$. superba Conti; некоторые виды отмечены в качестве новинок для отдельных районов. В то же время, перечислены и прокомментированы ошибочные указания представителей семейства для Монголии или определенных ее районов, обнародованные в основном после выхода «Конспекта ...» И.А. Губанова (1996). Валидизированы новые комбинации в родах Guenthera Andrz. и Stevenia Fisch. Сообщение может считатья альтернативной обработкой крестоцветных для недавно вышедшего «Конспекта сосудистых растений Монголии» (2014).

Since long time Mongolia attracts scientists from different countries focusing on biodiversity studies. More or less regular floristic investigations on the territory of the country are being conducted since 1830ths (Grubov, 1955; Gubanov, Hilbig, 1989; Gubanov, 1996) continuously bringing new data on the diversity of Mongolian plants. The basic and most referenced treatments of last decades are those of Grubov (1982) and Gubanov (1996) which are 
very useful as the key points reflecting the state of the knowledge on the flora of Mongolia at the end of XX century and still covering considerable portion of floristic diversity of the country and mirrowing main peculiarities of its flora. At the same time, intensive field and herbarium studies of last years yielded so considerable amount of new data (summarized in Urgamal et al., 2013) that the necessity of preparing an updated checklist became absolutely evident which was done by Mongolian colleagues shortly afterwards (Urgamal et al., 2014). It is enough to mention that the new conspectus included 3127 species and subspecies of vascular plants compared to 2823 in Gubanov (1996), i. e., ca. 11\% in addition, to demonstrate how important and timely work it has become.

Being a reflection of the current stage of knowledge on the floristic diversity and, often, taxa distribution of certain area, checklists represent the starting point for further studies in this field. Therefore, it is critical for such compilations to be maximally complete, up-to-date, and precise. The present communication was prompted by critical evaluation of the data on Cruciferae B. Juss. (Brassicaceae Burnett) - one of the leading families in Mongolian flora - presented in the new conspectus. According to Urgamal et al. (2014), it is the $5^{\text {th }}$ biggest family in the country with 61 genera and 160 species, covering over $5 \%$ of the floristic diversity of Mongolia. Hence, adequate information on the diversity of Cruciferae is essential for reliability of the whole checklist. On one hand, the treatment of the family in the new conspectus takes into consideration most of the updates published during last two decades and reflects apparent progress in studies of both species diversity and distribution of Mongolian mustards compared to Gubanov (1996). On the other hand, some discrepancies and misinterpretations of the previously published data distort the whole picture and need to be corrected; most important of them are the following.

1. Generic delimitation and assignment of certain species appears in some cases rather messy. For instance, among three very closely related species of Ptilotrichum C. A. Mey., nowadays assigned by some authors to Stevenia Fisch. (Stevenia canescens, $S$. dahurica, and $S$. tenuifolia), the latter is treated as a member of Alyssum L. while other two - as representatives of Ptilotrichum. Similarly, one of the two Mongolian Noccaea Moench species, $N$. ferganensis, is given under this generic name while another species, $N$. thlaspidioides, is attri- buted to Thlaspi L. as Th. cochleariforme although, like in previous case, both taxa are tightly related and often confused. The genus Strigosella Boiss. is accepted as distinct from Malcolmia W. T. Aiton but only one of the two species, $S$. brevipes, is given as Strigosella while another one, S. africana, is reported as Malcolmia. In the same way, the genus Oreoloma Botsch. is accepted as distinct from Sterigmostemum $\mathrm{M}$. Bieb., but very closely related to, if not conspecific with $O$. violaceum taxon is given as $S$. regeliorum and not as homotypic $O$. sulphureum. Listing Erysimum flavum under the generic name Hesperis L. is just beyond discussion.

2. Information on the species proven to be erroneously reported for Mongolia is not taken into account at all. In particular, all eight such species explicitly mentioned by German and Oyuntsetseg (2008) are included into the conspectus (with references to the publications where those species are excluded from Mongolian flora). Some further, most recent records (e. g., Arabis amplexicaulis, Erysimum ledebourii, E. quadrangulum) are based on various misinterpretations and cannot be confirmed.

3. Not all taxa reported as additions to Gubanov's "Conspectus ..." are in fact novelties but were included there under other names. These are Alyssum linifolium and Draba lanceolata given in Gubanov (1996) as Meniocus linifolius and Draba cana auct., respectively.

4. Information on loci classici of narrowly distributed species as well as assignment of certain taxa to endemics and subendemics is often inaccurate. For example, the following taxa are mentioned as Mongolian endemics, although none of them actually are: Crucihimalaya rupicola (said to be described from Gobi-Altai, in fact from Russian Altai); Galitzkya potaninii (occurs in neighboring Xinjiang; described from E Tian Shan); Microstigma brachycarpum (said to be described from "Transaltai Altai" [Transaltai Gobi], in fact from central Gansu). In cases of Crucihimalaya rupicola and Microstigma brachycarpum, indicated areas are type localities of their synonyms, C. mongolica and M. junatovii, respectively. Reported subendemics of Mongolia included some widely distributed taxa which at any rate (bearing in mind that definition of subendemic is rather relative) could not be treated as such, e. g.: Dendroarabis fruticulosa (distributed from Afghanistan through Middle Asia and Kazakhstan to NW China, W \& N Mongolia, SW and SE Siberia); Dontostemon micranthus (except Mongolia, also W, Central \& E Siberia, Far East, NW, N \& NE China); Draba altaica (Tibet, Himalayas, Pamir Alai, Tian 
Shan, Altai; one of the most widely distributed Asian alpine to subalpine Draba L. species); D. mongolica (N, NE \& Central China, W \& E Siberia, Far East, Mongolia); Erysimum quadrangulum (Central and E Europe, N Kazakhstan, SW Siberia, not occurring in Mongolia); Eutrema altaicum (mts. of S Kazakhstan, Kyrgyzstan, Tajikistan, W China (four provinces), and SW Siberia, not found in Mongolia); E. edwardsii (subcircumpolar and common in mts. of N Asia); Goldbachia pendula (Kazakhstan, Middle Asia, five western provinces of China, W Mongolia), Sisymbrium brassiciforme (Himalayas, Tibet, Pamir Alai, Tian Shan, southern part of Altai Mts.), common Irano-Turanian elements Neotorularia brevipes and Isatis multicaulis, etc. At the same time, true Mongolian endemic Dontostemon gubanovii is reported as subendemic; similarly, Rhammatophyllum kamelinii, if considered distinct from $R h$. erysimoides (as done in the treatment) is not found outside W Mongolia. Examples of omitted subendemics are Dontostemon perennis, Microstigma deflexum or Stevenia sergievskajae, all having, except Mongolia, rather limited distribution in neighboring S Siberia (D. perennis also known from one gathering in Xinjiang). Location of the type material is given for 12 arbitrarily selected taxa out of the total of 38 assigned to endemics and subendemics and, for some reason, for one species not treated as endemic/subendemic - Dontostemon dentatus ("typus MW"). In fact, no original material of this species is available at MW: lectotype of $D$. dentatus is in $\mathrm{P}$ and isolectotype in LE (German, 2012b).

5. Finally, distribution of some taxa in Mongolia (e. g., Chorispora bungeana, Crucihimalaya rupicola, Dontostemon elegans) is not accurately reported and needs to be corrected, in several cases just back to the information given by Gubanov (1996).

Taking into consideration the above concerns, it became evident that the data on Mongolian mustards presented in Urgamal et al. (2014) require correction. Hence, the main goal of this communication is providing the improved information on taxonomic diversity and distribution of representatives of this family in the country. Besides, it turned to be a good option to publish some floristic novelties including one state record, Matthiola superba, representing a new genus to the flora of Mongolia, and provide several taxonomic adjustments. For this purpose, available data were screened and critically evaluated including hebarium material from ALTB, BP, GAT, HAL, LE, MW, OSBU, PR, and TK; some images from UBA and UBU; online database "Flora
GREIF" (University of Greifswald ..., 2010 [continuously updated]; Rilke, Najmi, 2011; Rilke et al., 2012; Zemmrich et al., 2013) as well as relevant literature reports. The work is divided onto four parts: 1) conspectus itself with actual data on distribution of taxa; 2) enumeration of taxa orrurence of which either in Mongolia or in certain regions of the country was not confirmed; 3) floristic additions including one state and several regional records and 4) taxonomis remarks. In order to provide better comparison to the previous data, the way of presenting the information and basic symbols are the same as in the key reference point, i. e., Gubanov (1996), and in Urgamal et al. (2014). In particular, the taxa are arranged in the list alphabetically, for each species and subspecies an accepted name in italisized boldface is provided along with the basyonym and basic synonyms (mainly referring to Mongolia), all with nomenclatural references. For the convenience, all names accepted in the checklists of 1996 and 2014 but not in the current one (because of either being treated as synonyms or proven to be misapplied to Mongolian plants), are also incorporated (italized) and supplied, after the sign "=" or " $\equiv$ ", with a reference to accepted (hetero- or homotypic, respectively), non-highlighted name. Explanation of mistreatments (taxa sensu auct. fl. Mong., non orig.) are presented in the second part of this communication.

Distribution of species is given according to 16 number-coded phytogeohraphic regions of Mongolia elaborated by Grubov and Yunatov (1952) and subsequently corrected by Grubov (1963) and Gubanov et al. (1996) which are traditionally used in floristic works on Mongolia including Gubanov (1996) and Ugramal et al. (2014): 1 - Khubsugul (Hovsgol); 2 - Khentei; 3 - Khangai; 4 - MongolDaurian; 5 - Great Khingan; 6 - Khobdo (Khovd); 7 - Mongolian Altai; 8 - Middle Khalkha; 9 - East Mongolia; 10 - Depression of Great Lakes; 11 Valley of Lakes; 12 - East Gobi; 13 - Gobi-Altai; 14 - Dzungarian Gobi; 15 - Transaltai Gobi; 16 Alashan Gobi. The following signs indicate certain changes/updates compared to the previously published data:

"+" - addition after 1996 (before species/subspecies name - for Mongolia; after the number of phytogeographical region - for relevant region);

"\#" - change of the taxon name compared to Gubanov (1996).

All updates of the distribution (country and regional records) are supplied with references to the sources where those updates were first reported; in case of nomenclatural changes, references are 
provided to some recent publications introducing or proving relevant changes. Besides, number codes of records not given in Urgamal et al. (2014) (overlooked/misinterpreted or added in the present work) are given in boldface. Finally, like in previous checklists, emdemics and subendemics are specified; classical locatity and location of the type (holoor lectotype) is reported for such taxa.

The second part of the article is focused on erroneous data on distribution of Cruciferae species in Mongolia. Relevant information is embedded in two tables, one enumerating state and another - regional records. In all cases references to both publications reporting occurrence of taxa in the country or some of its phytogeographical regions and those claiming the contrary are provided. Whenever possible, primary sources of records are referred to; otherwise, any of the key treatments (Grubov, 1955, 1982; Gubanov, 1996) are cited which in some cases are also the primary sources of relevant floristic data. Only information on taxa wrongly reported for Mongolia or some of its regions in Gubanov (1996) or published later is taken into consideration as long as previous mistakes and discrepancies are corrected in earlier works (e. g., Grubov, 1955, 1972, 1982, etc.). Brief notes are provided for each entry.

The third part includes detailed documentation of new floristic records mentioned in the first part just with the sign "+" and reference "this study". In few cases, noteworthy accessions not representing regional records are discussed in addition. Finally (part four), several taxonomic adjustments are also provided.

Despite the elucidating character of publication, the author is free from illusion that all possible gaps/mistakes are filled in/improved here and, furthermore, that this work itself does not introduce some further unintended/overlooked discrepancies or arguable taxonomic decisions, for which I beg readers' pardon. However, I believe that it mostly improves available data rather than distorts them and provides most adequate picture of the current knowledge on taxonomic diversity and distribution of the Cruciferae of Mongolia.

\section{Conspectus of Mongolian Cruciferae (Brassicaceae)}

The present conspectus includes 141 species and 7 subspecies from 59 genera which is comparable with other recent estimations, e. g., 139 species and 5 subspecies from 55 genera by German \& Oyuntsetseg (2008) or 142 species from 58 genera by Rilke et al. (2012), but more sufficiently differs from 160 species [actually 159 species and one subspecies] from 61 genera by Urgamal et al. (2014) vs. 135 species and one subspecies from 58 genera reported by Gubanov (1996).

Alyssum desertorum Stapf $=$ A. turkestanicum

Alyssum lenense Adams, 1817, Mém. Soc. Nat. Mosc. 5: 110. - 1, 2, 3, 4, 5, 7, 8 , 9.

Alyssum linifolium Steph. ex Willd. $\equiv$ Meniocus linifolius

Alyssum obovatum (C. A. Mey.) Turcz. $\equiv$ Odontarrhena obovata

Alyssum tenuifolium Steph. ex Willd. $\equiv$ Stevenia tenuifolia (German, 2010)

\# Alyssum turkestanicum Regel et Schmalh., 1881, Izv. Imp. Obsc. Ljubit. Estestv. Moskovsk. Univ. 34, 2 [Descr. Pl. Nov. Rar. Fedtsch.]: 6. - A. desertorum Stapf, 1886, Denkschr. Acad. Wiss. (Math.-Naturw., Wien.), 51:302.-3, 6, 7, 10 .

Aphragmus involucratus (Bunge) O. E. Schulz, 1924, in Engler, Pflanzenreich, 86 (4, 105): 198. - Platypetalum involucratum Bunge, 1836, Enum.: 58. - 7, 13.

Arabidopsis mollissima (C. A. Mey.) N. Busch $\equiv$ Crucihimalaya mollissima (Al-Shehbaz et al., 1999).

Arabidopsis thaliana (L.) Heynh., 1842, in Holl et Heynh., F1. Sachs. 1: 538. - Arabis thaliana L., 1753, Sp. Pl. 2: 665. - 6, 7.

\# Arabis borealis Andrz. ex C. A. Mey., 1831, in Ledeb., Fl. Alt. 3: 25. - A. sagittata auct. non (L.) Scop.A. sagittata auct. non (Bertol.) DC. - 2, 3, 4, 5, 9.

Arabis amplexicaulis auct. non Edgew. $=$ Erysimum cheiranthoides

Arabis fruticulosa C. A. Mey. $\equiv$ Dendroarabis fruticulosa (German, Al-Shehbaz, 2008)

Arabis mongolica Botsch. $=$ Crucihimalaya rupicola (German, Ebel, 2005).

Arabis pendula L. $\equiv$ Catolobus pendulus (Al-Shehbaz, 2005).

Arabis rupicola Kryl. $\equiv$ Crucihimalaya rupicola (German, Ebel, 2005)

Arabis sagittata auct. non (Bertol.) DC. [A. hirsuta auct. non (L.) Scop.] = A. borealis (Knapp, Sančir, 1986; Knjasev, 2010) 
Barbarea arcuata (Opiz ex J. Presl et C. Presl) Reichenb. = B. vulgaris

Barbarea orthoceras Ledeb., 1824, Index Sem. Horti Dorpat.: 2. - Barbarea stricta auct. non Andrz. $2+$ (this study), 3, 4, 5, 9 .

Barbarea stricta auct. non Andrz. $=$ B. orthoceras (German, 2009)

\# Barbarea vulgaris W. T. Aiton, 1812, Hort. Kew., ed 2, 4: 109. - B. arcuata (Opiz ex J. Presl et C. Presl) Reichenb., 1822, Flora (Regensb.) 5 (1): 296. - 2, 3, 4, 5, 7.

+ Berteroa incana (L.) DC., 1821, Reg. Veg. Syst. Nat. 2: 291. - Alyssum incanum L., 1753, Sp. P1. 2: 650. $-2+$ (Doronkin et al., 2015; referred as to "Doronkin et al., 2012" in Oyuntsetseg, 2014), 4+ (Neuffer et al., 2012).

Brassica campestris L., 1753, Sp. P1. 2: 666. - B. rapa auct. non L. - 2, 3, 4, 6, 7, 8+ (Hilbig et al., 2012), 11.

Brassica juncea (L.) Czern., 1859, Consp. Pl. Charc.: 8. - Sinapis juncea L., 1753, Sp. P1. 2: 668. - 3, 4, 7, 9, 10, 11, 13, 14, 15+ (Hilbig, Tungalag, 2006).

Brassica rapa auct. non L. $=$ B. campestris

Note. Brassica oleracea L. is known exclusively in culture in Mongolia.

Braya glabella auct. non Richardson $=$ B. siliquosa

\# Braya humilis (C. A. Mey.) B. L. Rob., 1895, in A. Gray et S. Watson, Syn. Fl. N. Amer. 1 (1): 141. Sisymbrium humile C. A. Mey., 1830, in Ledeb., Icon. P1. Fl. Ross. 2: 16. - Torularia humilis (C. A. Mey.) O. E. Schulz, 1924, in Engler, Pflanzenreich, 86 (4, 105): 223. - Neotorularia humilis (C. A. Mey.) Hedge et J. Léonard, 1986, Bull. Jard. Bot. Natl. Belg. 56: 394. - T. grubovii Botsch., 1975, Bot. Zhurn., 60 (7): 948. - N. grubovii (Botsch.) Botsch., 1988, Bot. Zhurn. 73 (8): 1187. - N. mongolica Botsch. et Gubanov, 1988, Bot. Zhurn. 73 (8): 1186. - N. maximowiczii auct. non (Botsch.) Botsch. - 1, 3, 4, 6, 7, 8.

Braya rosea Bunge, 1839, Del. Sem. Horti Dorpat.: 7. - 1, 3, 6, 7.

Braya siliquosa Bunge, 1839, Del. Sem. Horti Dorpat.: 7. - B. glabella auct. non Richardson. -1 .

Bunias cochlearioides Murr. $\equiv$ Leiocarpaea cochlearioides (German, Al-Shehbaz, 2010)

Bunias orientalis L., 1753, Sp. Pl. 2: 670. - 9.

Camelina microcarpa Andrz. ex DC., 1821, Reg. Veg. Syst. Nat. 2: 517. - 4, 14+ (German, 2009).

Camelina sativa (L.) Crantz, 1762, Stirp. Austr. 1: 17. - Myagrum sativum L., 1753, Sp. Pl. 2: 641 - 4. Probably nowadays $C$. sativa does not occur in Mongolia.

Capsella bursa-pastoris (L.) Medik., 1792, Pflanzengatt. 1: 85. - Thlaspi bursa-pastoris L., 1753, Sp. Pl. 2: 647. - 2+ (Dulamsuren, Mühlenberg, 2003), 3, 4, 6, 7, 10, 14.

+ Capsella orientalis Klok., 1926, Byull. Obshch. Estestvoisp. Voronezhsk. Gosud. Univ. 1, 2-4: 122. - C. bursa-pastoris subsp. orientalis (Klok.) Tzvel., 2000, Novit. Syst. Pl. Vasc. 32: 184. - 3+ (this study), 7+ (German, Ebel, 2009), 10+ (this study).

Cardamine bellidifolia L., 1753, Sp. Pl. 2: 654. - 1, 2, 3, 7.

+ Cardamine impatiens L., 1753, Sp. P1. 2: 655. - 6+ (German, 2009).

Cardamine leucantha (Tausch) O. E. Schulz, 1903, Bot. Jahrb. Syst. 32 (2-3): 403. - Dentaria leucantha Tausch, 1836, Flora 19 (2): 404. -5.

Cardamine macrophylla Willd., 1800, Sp. Pl. 3 (1): 484. - 1, 2+ (Doronkin et al., 2015; referred as to "Khan, 2012" in Oyuntsetseg, 2014), 6, 7.

Cardamine parviflora L., 1759, Syst. Nat. ed. 10, 2: 1131. - 2, 3.

Cardamine pratensis L., 1753, Sp. Pl. 2: 656. - 1, 2, 3, 4, 5, 7, 9.

Cardamine prorepens Fisch. ex DC., 1821, Reg. Veg. Syst. Nat. 2: 256. - 5.

Cardamine trifida (Lam. ex Poir.) B. M. G. Jones, 1964, Feddes Repert. 69: 57. - Dentaria trifida Lam. ex Poir., 1812, Encycl. Meth. Bot. Suppl. 2: 465. - Sphaerotorrhiza trifida (Lam. ex Poir.) A. Khokhr., 1985, Fl. Magadansk. Obl.: 235. -5.

Cardaria pubescens (C. A. Mey.) Jarm. $\equiv$ Lepidium appelianum (Al-Shehbaz et al., 2002).

\# Catolobus pendulus (L.) Al-Shehbaz, 2005, Novon, 15 (4): 521. - Arabis pendula L., 1753, Sp. Pl. 2: 665. - 1, 2, 3, 4, 5, 6, 9, 10+ (this study), 12, 13.

Carpoceras ceratocarpum (Pall.) N. Busch $\equiv$ Thlaspi ceratocarpum (Meyer, 1973, 2001).

Chorispora bungeana Fisch. et C. A. Mey., 1841, Enum. P1. Nov. 1: 96. - 7.

Chorispora sibirica (L.) DC., 1821, Reg. Veg. Syst. Nat. 2: 437. - Raphanus sibiricus L., 1753, Sp. Pl. 2: 669. - 6, 7, 13, 14 . 
Chorispora tenella (Pall.) DC., 1821, Reg. Veg. Syst. Nat. 2: 435. - Raphanus tenellus Pall., 1776, Reise 3: 741. - 7, 14.

Clausia aprica (Steph. ex Willd.) Korn.-Tr. 1834, Index Sem. Horti Kazan.: sine pag. - Cheiranthus apricus Steph. ex Willd., 1800, Sp. P1. 3: 518. - Dontostemon apricus (Steph. ex Willd.) D. A. German, 2010, Komarovia 6 (2): 85. - 1, 2, 3, 4, 6, 7, 9, 13.

Clausia trichosepala (Turcz.) Dvořák, 1966, Phyton (Horn) 11: 200. - Hesperis trichosepala Turcz., 1832, Bull. Soc. Nat. Mosc. 5: 180. - 4.

Conringia planisiliqua Fisch. et C. A. Mey., 1837, Index Sem. Horti Bot. Petrop. 3: 32. - 14.

\# Crucihimalaya mollissima (C. A. Mey.) Al-Shehbaz, O'Kane et Price, 1999, Novon, 9 (3): 299. - Sisymbrium mollissimum C. A. Mey., 1831, in Ledeb., Fl. Alt. 3: 140. - Arabidopsis mollissima (C. A. Mey.) N. Busch, 1913, Fl. Sibir. Orient. Extr. 1: 136. - 6, 7, 13, 14.

\# Crucihimalaya rupicola (Kryl.) A. L. Ebel et D. A. German, 2005, Turczaninowia 8, 3: 8. - Arabis rupicola Kryl., 1902, Acta Horti Petrop. 21, 1: 3. - Arabidopsis rupicola (Kryl.) A. L. Ebel, 2000, Turczaninowia 3, 3: 19. - Arabis mongolica Botsch., 1975, Bot. Zhurn. 60 (7): 947. - Arabidopsis mongolica (Botsch.) Měsiček et Soják, 1995, Folia Geobot. Phytotax. 30: 445. - Arabidopsis mongolica (Botsch.) A. L. Ebel, 2000, Turczaninowia 3, 3: 19, comb. superfl. - Crucihimalaya mongolica (Botsch.) Al-Shehbaz, O'Kane et Price, 1999, Novon 9 (3): 300. - 6+ (German, 2009), 7, 10, 11, 13, 14. - Subendemic; described from SW Siberia (SE Altai); lectotype (Polozhij, Balashova, 1989) - TK, isolectotypes - LE, TK.

\# Dendroarabis fruticulosa (C. A. Mey.) D. A. German et Al-Shehbaz, 2008, Harvard Pap. Bot. 13 (2): 290. - Arabis fruticulosa C. A. Mey., 1831, in Ledeb., Fl. Alt. 3: 19. - Rhammatophyllum fruticulosum (C. A. Mey.) Al-Shehbaz, 2005, Novon, 15 (4): 522. - 1+ (Hayasaka, 2011, as Arabis fruticulosa), 7.

Descurainia sophia (L.) Webb ex Prantl, 1891, in Engler und Prantl, Nat. Pflanzenfam. 3, 2: 192. - Sisymbrium sophia L., 1753, Sp. P1. 2 : 659. - 1, 2, 3, 4, 5, 6, 7, 8, 9, 10, 12, 13, 14.

Dimorphostemon pectinatus (DC.) V. Golubk. $\equiv$ Dontostemon pinnatifidus (Al-Shehbaz, Ohba, 2000).

Dontostemon crassifolius Bunge, 1858, in Maxim., Prim. Fl. Amur.: 46. - 7, 10, 12, 13, 16. - Subendemic; described from East Gobi phytogeographical region; lectotype (Buzunova, 2000) - LE, isolectotypes $-\mathrm{K}, \mathrm{P}$.

+ Dontostemon dentatus (Bunge) Ledeb., 1841, Fl. Ross. 1: 145. - Andreoskia dentata Bunge, 1833, Enum. Pl. China Bor.: 6. - 5+ (Gubanov, 1999).

Dontostemon elegans Maxim., 1889, Enum. Pl. Mong.: 57. - 7, 11, 13, 14, 15, 16. - Subendemic; described from Chinese Dzungaria and SW Mongolia; lectotype from Mongolian Altai at the border with Transaltai Gobi phytogeographical region (Buzunova, 2000) - LE.

+ Dontostemon gubanovii (D. A. German) D. A. German, 2010, Komarovia 6 (2): 85. - D. senilis subsp. gubanovii D. A. German, 2007, Novon, 17 (2): 173. - 6+, 7+, 10+ (German, 2007). - Endemic; described from Khobdo phytogeographical region (lake Achit-Nuur); holotype - MW.

Dontostemon integrifolius (L.) C. A. Mey., 1831, in Ledeb., Fl. Alt. 3: 120. - Sisymbrium intergifolium L., 1753, Sp. P1. 2 : 660. - 1, 2, 3, 4, 5, 6, 7, 8, 9, 10, 11, 12, 13, 16.

Dontostemon micranthus C. A. Mey., 1831, in Ledeb., Fl. Alt. 3: 120. - Sisymbrium lineare DC., 1821, Reg. Veg. Syst. Nat. 2: 464, nom. rej. prop. (German, 2012d). - 1, 2, 3, 4, 5+ (German, 2009), 8, 9, 13.

Dontostemon perennis C. A. Mey., 1831, in Ledeb., Fl. Alt. 3: 121. - 3, 6, 7, 10, 11, 13. - Subendemic; described from SW Siberia (SE Altai); lectotype (German, 2005a) - LE, isolectotypes - B, BP, GOET, HAL, KW, LE, M, P, W.

\# Dontostemon pinnatifidus (Willd.) Al-Shehbaz et H. Ohba, 2000, Novon, 10 (1): 96. - Cheiranthus pinnatifidus Willd., 1800, Sp. P1. 3: 523. - Dimorphostemon pinnatifidus (Willd.) H. L. Yang, 2009, J. Desert. Res. 29 (3): 435. - Sisymbrium pectinatum DC., 1821, Reg. Veg. Syst. Nat. 2: 485, nom. illeg. superfl. Dimorphostemon pectinatus (DC.) V. Golubk., 1974, Bot. Zhurn. 59 (10): 1453, comb. illeg. - 1, 4, 8, 13.

Dontostemon senilis Maxim., 1880, Bull. Acad. Sci. Pétersb. 26: 421. - 6, 7, 10, 11, 12, 13, 14, 15, 16. - Subendemic; described from China (Ordos and East Tian Shan); lectotype from Ordos (Grubov in Buzunova, 2000) - LE.

Draba altaica (C. A. Mey.) Bunge, 1841, Del. Sem. Horti Dorpat.: 8. - D. rupestris R. Br. [var.] $\beta$ altaica C. A. Mey., 1831, in Ledeb. Fl. Alt. 3: 72. - 6, 7, 13+ (German, 2009; misprinted as "10+" in Oyuntsetseg, 2014). 
Draba baicalensis Tolm., 1939, Fl. URSS 8: 650. - D. czuensis Revuschk. et A. L. Ebel, 1998, Animadv. Syst. Herb. Kryl. Univ. Tomsk. 90: 4. - 3, 6+ (Ebel, 2002; Ebel, Rudaya, 2002, both times as D. czuensis), 7. - Subendemic; described from SE Siberia (lake Baikal); holotype - LE.

Draba cana auct. non Rydb. = D. lanceolata

Draba czuensis Revuschk. et A. L. Ebel = D. baicalensis

Draba denserosulata Pobed. $=$ D. lanceolata (German, 2008)

Draba eriopoda Turcz. ex Ledeb., 1841, Fl. Ross. 1: 154. - 1, 2, 3, 6.

Draba fladnizensis Wulfen, 1779, in Jacq., Miscell. Austr. Bot. 1: 147. - 1, 2, 3, 6, 7, 13.

Draba hirta L., 1759, Syst. Nat. ed. 10, 2: 1127. - D. glabella Pursh, 1814, Fl. Amer. Sept. 2: 434. - 1,

$2,3,6,7,13$.

The name $D$. hirta "appears to have been widely, though informally, rejected as confused or ambiguous" (The Linnaean Plant Name Typification Project: http://www.nhm.ac.uk/research-curation/research/projects/linnaean-typification/) as "the material at LINN is in bad condition and probably belongs to two species" (Al-Shehbaz et al., 2010). Instead, the binominal D. glabella is being often in use (e. g., Walters et al., 1993; Al-Shehbaz et al., 2010). On the other hand, there is no common practice of application of the name $D$. hirta in any other sense and it is persistently used in floristic literature, especially dealing with Northern Eurasia including all Mongolian treatments. Therefore, unless formal nomenclatural actions preventing further application of the name $D$. hirta are undertaked, it should be used in the current sense as having priority for this species.

Draba kusnetzowii (Turcz. ex Ledeb.) Hayek, 1911, Beih. Bot. Centralbl. 27, 1: 172. - Holargidium kusnetzowii Turcz. ex Ledeb., 1841, Fl. Ross. 1: 156. - 1, 3, 6, 7, 13.

\# Draba lanceolata Royle 1839, Illustr. Bot. Himal. Mts. 1: 72. - Draba denserosulata Pobed., 1935, Acta Mong. Comm. Acad. Sci. USSR 19: 55. - D. cana auct. non Rydb. - 1, 2, 3, 4, 6, 7, 13.

Draba mongolica Turcz., 1842, Bull. Soc. Nat. Mosc. 15, 2: 256. - 1, 3.

Draba nemorosa L., 1753, Sp. P1. 2 : 643. - 1, 2, 3, 4, 5, 6, 7, 8, 9, 10, 13.

Draba ochroleuca Bunge, 1835, Mém. Sav. Etr. Pétersb. 2: 574. - 1, 2, 3, 6, 7.

Draba oreades Schrenk, 1842, in Fisch. et C. A. Mey., Enum. Pl. Nov. 2: 56. - 1, 3, 6, 7, $13+$ (German, 2009).

Draba pygmaea Turcz. ex N. Busch, 1918, Izv. Rossijsk. Akad. Nauk, ser. 6, 12: 1633, 1639. - 1, 3. Subendemic; described from South-East Siberia (E Sayan and Transbaikal area); lectotype from Nuchu-Daban (German, 2011a) - LE, isolectotypes - K, KW, LE, MW.

Draba sibirica (Pall.) Thell., 1906, Mitt. Bot. Mus. Zürich 28: 318. - Lepidium sibiricum Pall., 1776, Reise 3: 34. - 3, 7.

+ Draba stenocarpa Hook. fil. et Thoms., 1861, Journ. Proc. Linn. Soc. Bot. 5: 153. - 7+ (German, 2001).

Draba subamplexicaulis C. A. Mey., 1831, in Ledeb., Fl. Alt. 3: 77. - 1, 2, 3, 6, 7, 13.

Draba turczaninowii Pohle et N. Busch, 1918, Izv. Rossijsk. Akad. Nauk, ser. 6, 12: 1633. - 1+ (German, 2009), 6+ (Ebel, 2002; Smirnov et al., 2003), 7, 13+ (German, 2009).

Eruca sativa Mill., 1768, Gard. Dict. ed. 8, no. 1. - E. vesicaria auct. non (L.) Cav. - 4, 6, 7, $11,14$.

Eruca vesicaria auct. non (L.) Cav. $=$ E. sativa

Erucastrum armoracioides (Czern. ex Turcz.) Cruchet $=$ Guenthera persica

\# Erysimum andrzejowskianum Bess. ex DC., 1821, Reg. Veg. Syst. Nat. 2: 502. - E. diffusum auct. non Ehrh. - E. canescens auct. non Roth -7 .

Erysimum canescens auct. non Roth $=$ E. andrzejowskianum

Erysimum cheiranthoides L., 1753, Sp. P1. 2 : 661, s. str. - 1, 2, 3, 4, 5, 7, 8, 9, 10, 11, 13, 14.

+ Erysimum cheiranthoides subsp. altum Ahti, 1961, Arch. Soc. Zool. Bot. Fenn. (Arch. Soc. "Vanamo"), 16, 1: 24. - E. altum (Ahti) Tzvel., 1997, Bot. Zhurn. 82, 12: 94. - E. cheiranthoides auct. non L., p. p. - ? (Ahti, 1961, without indication of regions or localities).

+ Erysimum cheiranthoides subsp. transiliense (M. Pop.) D. A. German, 2006, Animadv. Syst. Herb. Kryl. Univ. Tomsk. 97: 26. - E. transiliense M. Pop., 1940, Not. Syst. Herb. Inst. Bot. Acad. Sci. URSS 8: 74. - E. virgatum auct. non Roth, p. p. - 7+ (German, 2006).

Erysimum flavum (Georgi) Bobrov, 1960, Not. Syst. Herb. Inst. Bot. Acad. Sci. URSS 20: 15. - Hesperis flava Georgi, 1775, Bemerk. Reise Russ. Reich 1: 225. - 1, 2, 3, 4, 5, 8, 9, 12+ (Darijmaa et al., 2004; cited from: Oyuntsetseg, 2014), 13.

+Erysimum flavum subsp. altaicum (C. A. Mey.) Polozh. ex Doronkin, 1994, Fl. Sib. 7: 68. - E. altaicum C. A. Mey., 1831, in Ledeb., Fl. Alt. 3: 153. - E. humillimum (C. A. Mey.) N. Busch, 1939, Fl. URSS 
8: 106, 638. - E. altaicum var. humillimum C. A. Mey., 1831, in Ledeb., Fl. Alt. 3: 153. - E. flavum subsp. humillimum (C. A. Mey.) A. L. Ebel, 2000, Turczaninowia 3, 3: 27. - 3+, 6+, 7+, 10+ (Ebel, 2000a, as E. flavum subsp. humillimum; German, Oyuntsetseg, 2008).

Delimitation of subsp. altaicum and subsp. flavum is not always obvious based on Mongolian material. Geographically, the former is restricted to the western and the latter - to the central and eastern parts of the country.

Erysimum hieraciifolium auct. non L. $=$ E. marschallianum

+ Erysimum kotuchovii D. A. German, 2004, Turczaninowia, 7, 2: 14. - Syrenia macrocarpa auct. non Vass., p. p. - E. vassilczenkoi auct. non Polatschek, p. p. - E. quadrangulum auct. non Desf. $-7+$ (German, 2005b). - Subendemic; described from East Kazakhstan (valley of Bukhtarma); holotype - ALTB, isotype Herb. of Altai Botanical Garden, Ridder.

\# Erysimum marschallianum Andrz. ex M. Bieb., 1819, F1. Taur.-Cauc. 3: 441. - E. hieraciifolium auct. non L. - E. virgatum auct. non Roth, p. p. - 2, 3, 4, 6, 7, 10, 13, 14.

+ Erysimum mongolicum D. A. German, 2005, Willdenowia, 35 (2): 307. - Syrenia macrocarpa auct. non Vass., p. p. - E. vassilczenkoi auct. non Polatschek, p. p. - 7+, 14+ (German, 2005b). - Subendemic; described from Mongolian Altai phytogeographical region (valley of Bodonchijn-gol); holotype - ALTB, isotypes - B, LE, MO, OSBU.

Erysimum quadrangulum auct. non Desf. $=$ E. kotuchovii (German, 2012a)

Erysimum sisymbrioides C. A. Mey., 1831, in Ledeb., Fl. Alt. 3: 150. - 6, 7, 15.

Erysimum vassilczenkoi auct. non Polatschek $=$ E. kotuchovii et E. mongolicum (German, 2005b)

Erysimum virgatum auct. non Roth $=$ E. cheiranthoides subsp. transiliense and E. marschallianum

Eutrema altaicum (C. A. Mey.) Al-Shehbaz et S. I. Warwick - not in the flora (Ebel, 2000a, as Taphrospermum altaicum)

Eutrema edwardsii R. Br., 1823, Chloris Melvill.: 9, s. str. - 1, 2, 3, 6, 7, 13.

+ Eutrema edwardsii subsp. compactum (O. E. Schulz) A. L. Ebel, 2000, Turczaninowia 3, 3: 30. E. compactum O. E. Schulz, 1922, in W. Limpricht, Feddes Repert. 12: 387. - Braya heterophylla W. W. Smith, 1919, Notes Roy. Bot. Gard. Edinburgh 11: 201. - E. heterophyllum (W. W. Smith) H. Hara, 1973, J. Jap. Bot. 48: 97. - E. edwardsii subsp. heterophyllum (W. W. Smith) D. A. German, 2010, Komarovia 6 (2): 82. - 7+ (German, 2001, as E. heterophyllum).

Identity of Mongolian specimens reported as E. edwardsii subsp. compactum might require further elucidation.

\# Eutrema salsugineum (Pall.) Al-Shehbaz et S. I. Warwick, 2005, Harvard Pap. Bot. 10 (2): 134. - Sisymbrium salsugineum Pall., 1773, Reise 2: 740. - Thellungiella salsuginea (Pall.) O. E. Schulz, 1924, in Engler, Pflanzenreich, 86 (4, 105): 252. - 3, 4, 7, 8, 9, 10.

Galitzkya macrocarpa (Ik.-Gal.) V. Bocz., 1979, Bot. Zhurn. 64 (10): 1442. - Berteroa macrocarpa Ik.-Gal., 1936, Acta Inst. Bot. Acad. Sci. URSS, ser. 1, 3: 189. - Hormathophylla macrocarpa (Maxim.) Kamelin, 1973, Florogenet. Analysis Nat. Fl. Mount. Middle Asia: 267. - 13, 15. - Endemic; described from Gobi-Altai phytogeographical region (Bayan-Tsagan-Nuruu); holotype and isotype - LE.

Galitzkya potaninii (Maxim.) V. Bocz., 1979, Bot. Zhurn. 64 (10): 1442. - Berteroa potaninii Maxim., 1880, Bull. Acad. Sci. Pétersb. 26 (3): 422. - Hormathophylla potaninii (Maxim.) V. I. Dofor., 2012, Consp. Fl. Eur. Or. 1: 393. - 7, 14, 15. - Subendemic; described from China (Xinjiang, East Tian Shan); lectotype (Boczantzeva, 1979) - LE.

Goldbachia ikonnikovii Vass. - 6+ (Gubanov, 1996, misprinted as 9; German et al., 2003); 7+ (Revushkin et al., 2001); 8+, 10+ (German, 2009), 11, 13, 14+(German, 2009). - Subendemic; described from Gobi-Altai phytogeographical region (mt. Bayan-Boro-Nuruu); lectotype (Botschantzev, 1963) - LE, isolectotypes - LE, NS, TK.

Goldbachia laevigata auct. non (M. Bieb.) DC. = G. pendula (German, 2001) and G. ikonnikovii (German, 2009)

+ Goldbachia pendula Botsch., 1963, Not. Syst. Herb. Inst. Bot. Acad. Sci. URSS 22: 140. - 7+, 14+ (German, 2001).

\# Guenthera persica (Boiss. et Hohen.) D.A. German, 2015, Turczaninowia 18, 2: 62. - Brassica persica Boiss. et Hohen., 1849, Diagn. Pl. Or. Nov. 8: 26. - G. elongata (Ehrh.) Andrz. subsp. integrifolia (Boiss.) Gómez-Campo, 2003, Anales Jard. Bot. Madrid 60: 304. - B. elongata Ehrh. var. integrifolia Boiss., 1867, Fl. Or. 1: 394. - B. obtusangula (G. Haller ex Schleich.) Reichenb. subsp. integrifolia (Boiss.) 
Arcang., 1894, Comp. Fl. Ital., ed. 2: 267. - B. elongata subsp. integrifolia (Boiss.) Breistr., 1944, Bull. Soc. Sci. Dauphiné 60: 139. - Brassica armoracioides Czern. ex Turcz., 1854, Bull. Soc. Nat. Mosc. 27 (2): 311. - Erucastrum armoracioides (Czern. ex Turcz.) Cruchet, 1902, Bull. Soc. Vaud. Sci. Nat.: 333. - B. elongata subsp. armoracioides (Czern. ex Turcz.) Asch. et Graebn., 1898, Fl. Nordostdeut. Flachl.: 360. B. elongata auct. non Ehrh. - 4, 9.

Hesperis flava Georgi $\equiv$ Erysimum flavum (Bobrov, 1960; German, 2012c)

Hesperis matronalis auct. non L. $=\mathrm{H}$. sibirica

Hesperis sibirica L., 1753, Sp. Pl. 2: 663. - H. pseudonivea Tzvel., 1959, Not. Syst. Herb. Inst. Bot. Acad. Sci. URSS 19: 131. - H. sibirica subsp. pseudonivea (Tzvel.) A. L. Ebel, 2000, Turczaninowia 3, 3 : 32. - H. matronalis auct. non L. $-1,2,3,4,7$.

\# Hornungia procumbens (L.) Hayek, 1925, Feddes Repert. 30: 480. - Lepidium procumbens L., Sp. P1. 2: 643. - Hymenolobus procumbens (L.) Nutt., 1838, in Torr. et A. Gray, Fl. N. Amer. 1: 117. - 3, 6+ (Smirnov et al., 2003, as Hymenolobus procumbens), 7, 10, 11, 14.

Hymenolobus procumbens (L.) Nutt. $\equiv$ Hornungia procumbens (Appel, Al-Shehbaz, 1997)

Isatis costata C. A. Mey., 1831, in Ledeb., Fl. Alt. 3: 204. - I. tinctoria auct. non L. - 2, 3, 4, 6+ (German, 2009), 7, 8, 9, 11, 12, 13, 14.

\# Isatis gymnocarpa (Fisch. ex DC.) Al-Shehbaz, Moazzeni et Mummenhoff, 2012, Taxon 61 (5): 948, non I. lasiocarpa Ledeb., 1841. - Tauscheria gymnocarpa Fisch. ex DC., 1821, Reg. Veg. Syst. Nat. 2: 564. - T. lasiocarpa Fisch. ex DC., 1821, Reg. Veg. Syst. Nat. 2: 563. - 14.

+ Isatis multicaulis (Kar. et Kir.) Jafri, 1973, Fl. West Pakistan 55: 72. - Pachypteris multicaulis Kar. et Kir., 1842, Bull. Soc. Nat. Mosc. 15 (1): 159. - Pachypterygium multicaule (Kar. et Kir.) Bunge, 1843, Del. Sem. Horti Dorpat.: 8. - 14+ (Smirnov et al., 2003).

Isatis oblongata DC., 1821, Reg. Veg. Syst. Nat. 2: 471. - I. tinctoria L. subsp. oblongata (DC.) N. Busch, 1913, Fl. Sibir. Orient. Extr. 1: 156, 158. - 1, 3, 4, 8, 9, 13.

Isatis tinctoria auct. non L. $=$ I. costata (Smirnov et al., 2003)

\# Leiocarpaea cochlearioides (Murr.) D. A. German et Al-Shehbaz, 2010, Nordic J. Bot. 28 (6): 648. Bunias cochlearioides Murr. 1777, Novi Comment. Gotting. 8: 42. - 1.

Leiospora exscapa (C. A. Mey.) Dvořák, 1968, Spisy Prirod. Fak. Univ. Brno 497: 357. - Parrya exscapa C. A. Mey. 1829, in Ledeb., Icon. Pl. Fl. Ross. 1: 21. - 1, 6, 7.

Lepidium affine Ledeb., 1821, Index Sem. Horti Dorpat.: 22. - L. sibiricum Schweigg., 1812, Enum. Pl. Horti Regiomont.: 43, non Pall., 1776. - L. latifolium L. subsp. sibiricum Thell. 1906, Neue Denkschr. Schweiz. Ges. Naturw. 4: 161. - L. latifolium auct. non L., p. p. - 4, 7+ (Smirnov et al., 2003, as L. latifolium subsp. sibiricum Thell.), $8+$ (this study), 9, 14.

Lepidium amplexicaule Willd., 1800, Sp. P1. 3 (1): 436. - 3, 7, 8, 9?, 10, 11, 14, 15. - A record from 9 (Neuffer et al., 2012) is based on two specimens one of which (OSBU 20713) represents L. affine and another (OSBU 20481 ) is somewhat intermediate between the latter and $L$. amplexicaule though still closer to $L$. affine. It is likely that L. amplexicaule does not occur in East Mongolian region.

\# Lepidium apetalum Willd., 1800, Sp. Pl. 3 (1): 439. - L. densiflorum auct. non Schrad. - L. ruderale auct. non L. - 1, 2, 3, 4, 5, 6+ (this study), 7, 8, 9, 10, 11, 12, 13, 14, 15.

\# Lepidium appelianum Al-Shehbaz, 2002, Novon, 12 (1): 7, non L. pubescens Desv. (1815), nec Tineo (1827). - Hymenophysa pubescens C. A. Mey., 1830, in Ledeb., Icon. Pl. Fl. Ross. 2: 20. - Cardaria pubescens (C. A. Mey.) Jarm., 1934, Weed Fl. USSR 3: 29. - 7, 10, 11, 14, 15, 16.

\# Lepidium cartilagineum (J. Mayer) Thell., 1906, Viert. Naturf. Ges. Zürich 51: 173. - Thlaspi cartilagineum J. Mayer, 1786, Abh. Böhm. Ges. Wiss. (Math.-Nat.) 2: 235. - L. crassifolium Waldst. et Kit., 1799, Descr. Icon. Pl. Rar. Hung. 1: 4. - 5, 7+ (Revushkin et al., 2001, as L. crassifolium), 8, 10, 12, 14+ (Revushkin et al., 2001, as L. crassifolium).

Lepidium cordatum Willd. ex Steven, 1821, in DC. Reg. Veg. Syst. Nat. 2: 554. - 6, 7, 8, 9, 10, 11, 13, $14,15,16$.

Lepidium crassifolium Waldst. et Kit. $=$ L. cartilagineum

Lepidium densiflorum auct. non Schrad. = L. apetalum (Ebel, 2001)

Lepidium lacerum C. A. Mey., 1830, in Ledeb., Icon. P1. Fl. Ross. 2: 19. - L. songaricum auct. non Schrenk - 7+ (Revushkin et al., 2001), 14.

Lepidium latifolium auct. non L. $=$ L. affine and L. obtusum 
Lepidium obtusum Basin., 1844, Bull. Acad. Sci. Pétersb. 2: 203. - L. latifolium auct. non L., p. p. - 6, 7, 10, 14, 15 .

Lepidium ruderale auct. non $\mathrm{L} .=\mathrm{L}$. apetalum

Lepidium songaricum auct. non Schrenk = L. lacerum (German, 2009).

+Litwinowia tenuissima (Pall.) Woron. ex Pavl., 15 XII 1934, Fl. Centr. Kaz. 2: 302. - Vella tenuissima Pall., 1776, Reise 3: 780. - 14+ (Smirnov et al., 2003).

Macropodium nivale (Pall.) W. T. Aiton, 1812, Hort. Kew., ed. 2, 4: 108. - Cardamine nivalis Pall., 1773, Reise 2: 740. - 1, 7.

Malcolmia africana (L.) W. T. Aiton $\equiv$ Strigosella africana (Botschantzev, 1972; Al-Shehbaz et al., 2014)

+ Matthiola superba Conti, 1900, Mém. Herb. Boiss. 18: 23. - 14+ (this study).

Megacarpaea megalocarpa (Fisch. ex DC.) Schischk. ex B. Fedtsch., 1939, Fl. URSS 8: 543. - Biscutella megalocarpa Fisch. ex DC., 1811, Ann. Mus. Hist. Nat. 18: 296. - 14.

Meniocus linifolius (Steph. ex Willd.) DC., 1821, Reg. Veg. Syst. Nat. 2: 325. - Alyssum linifolium Steph. ex Willd., 1800, Sp. P1. 3 (1): 467. - 3, 7.

\# Microstigma brachycarpum Botsch., 1959, Bot. Zhurn. 44 (10): 1485. - M. junatovii Grub., 1978, Bot. Zhurn. 63 (3): 363. - 15, 16+ (Smirnov et al., 2003, as M. junatovii). - Subendemic; described from China (Gansu, Beidashan Mts. north of Jinchang); holotype - LE.

Microstigma deflexum (Bunge) Juz., 1939, Fl. URSS 8: 298. - Matthiola deflexa Bunge, 1839, Del. Sem. Horti Dorpat.: 8. - Sterigmostemum botschantzevii Grub., 1978, Bot. Zhurn. 63 (3): 363. - 3+ (Ebel, 2000a), 6, 7, 12, 13, 16+ (German, 2009). - Subendemic; described from SW Siberia (Central Altai); lectotype (German, 2005a) - LE, isolectotypes - B, G, HAL, LE, P.

Microstigma junatovii Grub. $=$ M. brachycarpum (Zhou et al., 2001)

+ Neotorularia brevipes (Kar. et Kir.) Hedge et J. Léonard, 1986, Bull. Jard. Bot. Nat. Belg. 56 (3-4): 393. - Sisymbrium brevipes Kar. et Kir. 1842, Bull. Soc. Nat. Mosc. 15 (1): 154, excl. var. - Malcolmia brevipes (Kar. et Kir.) Boiss., 1867, Fl. Or. 1: 267. - 7+ (Smirnov et al., 2003), 14+ (Maximowicz, 1889, as Malcolmia brevipes; Smirnov et al., 2003).

Neotorularia grubovii (Botsch.) Botsch. = Braya humilis (Al-Shehbaz, German, 2014)

Neotorularia humilis (C. A. Mey.) Hedge et J. Léonard $\equiv$ Braya humilis

Neotorularia maximowiczii auct. non (Botsch.) Botsch. = Braya humilis (German, 2001, as N. humilis)

Neotorularia mongolica Botsch. et Gubanov = Braya humilis (Al-Shehbaz, German, 2014)

Neotorularia korolkowii (Regel et Schmalh.) Hedge et J. Léonard - not in the flora (German, 2009)

Neslia paniculata (L.) Desv., 1815, Journ. Bot. Appl. (Paris) 3, 4: 162. - Myagrum paniculatum L., 1753, Sp. P1. 2: 641. - 2, 3, 4.

+ Noccaea ferganensis (N. Busch) Czer. 1981, Vasc. Pl. USSR: 140. - Thlaspi ferganense N. Busch, 1936, Bot. Journ. USSR 31 (5): 551. - T. cochleariforme auct. non DC., p. min. p. - 7+ (German, 2009).

\# Noccaea thlaspidioides (Pall.) F. K. Mey., 2010, Haussknechtia 12: 5 - Lepidium thlaspidioides Pall., 1776, Reise 3: 161. - Thlaspi cochleariforme DC., 1821, Reg. Veg. Syst. Nat. 2: 381. - N. cochleariformis (DC.) Á. Löve et D. Löve, 1975, Bot. Notiser 128: 513. - 1, 2, 3, 4, 5, 6, 7, 8, 9, 13.

\# Odontarrhena obovata C. A. Mey., 1831, in Ledeb., Fl. Alt. 3: 61. - Alyssum obovatum (C. A. Mey.) Turcz., 1837, Bull. Soc. Nat. Mosc. 10, 1: 57. - Alyssum microphylliforme Nyár., 1949, Anal. Acad. Rep. Popul. Române, Ser. A, 1(3): 11. - 1, 2, 3, 4, 5, 6, 7, 8, 9, 10.

+ Olimarabidopsis pumila (Čelak.) Al-Shehbaz, O'Kane et Price, 1999, Novon, 9 (3): 303. - Sisymbrium pumilum Steph. 1800, in Willd., Sp. Pl. 3: 507, nom. illeg., non Lam., 1779. - Stenophragma pumilum Čelak., 1872, Flora 55: 442. - Arabidopsis pumila (Čelak.) N. Busch, 1909, Fl. Cauc. crit. 3, 4: 465. - Thellungiella pumila (Čelak.) V. I. Dorof., 2002, Turczaninowia 5, 3: 110. - Calymmatium pumilum (Čelak.) D. A. German, 2010, Komarovia 6 (2): 86. - 14+ (Ebel, Rudaya, 2002, as Arabidopsis pumila).

Oreoloma violaceum Botsch. $\equiv$ Sterigmostemum violaceum (Yang, 1987; Kamelin, German, 2001)

Pachyneurum grandiflorum (C. A. Mey.) Bunge, 1839, Del. Sem. Horti Dorpat.: 8. - Draba grandiflora C. A. Mey., 1831, in Ledeb., Fl. Alt. 3: 74. - 1, 3, 6, 7, 10+ (Günther \& Schnittler in Flora GREIF: University of Greifswald ..., 2010+), 13. - Subendemic; described from SW Siberia (SE Altai); lectotype (German, 2005a) - LE, isolectotypes - B, GOET, LE, M, W.

Pachypterygium multicaule (Kar. et Kir.) Bunge $\equiv$ Isatis multicaulis (Jafri, 1973; Al-Shehbaz, 2012) 
Prionotrichon kamelinii Botsch. $=$ Rhammatophyllum erysimoides

Ptilotrichum canescens (DC.) C. A. Mey. $\equiv$ Stevenia canescens (Krasnoborov, German, 2007)

Ptilotrichum dahuricum Peschkova $\equiv$ Stevenia dahurica (German, Al-Shehbaz, 2010)

Ptilotrichum tenuifolium (Steph. ex Willd.) C. A. Mey. $\equiv$ Stevenia tenuifolia (German, 2010)

Pugionium dolabratum Maxim., 1880, Bull. Acad. Sci. Pétersb. 26: 426. - P. calcaratum Kom., 1932, Bull. Jard. Bot. Acad. Sci. URSS 30, 5-6: 718. - P. cristatum Kom., 1932, Bull. Jard. Bot. Acad. Sci. URSS 30, 5-6: 719. - 11, 12, 13, 16. - Subendemic; described from China (Inner Mongolia, Ordos); lectotype (Illarionova, 1999) - LE.

Pugionium pterocarpum Kom., 1932, Bull. Jard. Bot. Acad. Sci. URSS 30, 5-6: 720. - 10. - Subendemic; described from Depression of Great Lakes phytogeographical region (to the south of the lake Bayan-Nuur); lectotype (Illarionova, 1999) and isolectotypes - LE.

Yu et al. (2010) provided convincing evidences confirming previous finding of Illarionova (1999) regarding conspecificity of specimens from China (Inner Mongolia, Dengkou) described as P. dolabratum var. platypterum H. L. Yang and subsequently assigned to P. pterocarpum, with $P$. dolabratum. This fact, however, does not mean that plants of true $P$. pterocarpum from NW Mongolia and adjacent Russia (Tuva) should also be classified as $P$. dolabratum as suggested by Yu et al. (2010) who did not include Mongolian/Tuvinian specimens into their analyses (Yu et al., 2010, 2013). Unless it is done, suggested merge of P. pterocarpum with $P$. dolabratum cannot be accepted and based on the distinct morphological pattern (conf. Grubov, 1982; Illarionova, 1999) it is rather unlikely that conspecificity of P. pterocarpum and P. dolabratum might be proven.

Raphanus raphanistrum L., 1753, Sp. Pl. 2: 669. - 3, 4, 7, 8.

\# Rhammatophyllum erysimoides (Kar. et Kir.) Al-Shehbaz et O. Appel, III 2002, Novon, 12 (1): 3. Arabis erysimoides Kar. et Kir., 1842, Bull. Soc. Nat. Mosc. 15 (1): 145. - Rh. erysimoides (Kar. et Kir.) Kamelin, XII 2002, Bot. Zhurn. 87, 12: 108, comb. superfl. - Prionotrichon erysimoides (Kar. et Kir.) Botsch. et Vved., 1948, Not. Syst. Herb. Inst. Bot. Zool. Acad. Sci. Uzbek. 12: 8. - P. kamelinii Botsch., 1987, Novit. Syst. Pl. Vasc. 24: 98. - Rh. kamelinii (Botsch.) Al-Shehbaz et O. Appel, III 2002, Novon, 12 (1): 3. - Rh. kamelinii (Botsch.) Kamelin, XII 2002, Bot. Zhurn. 87, 12: 109, comb. superfl. - 7, 14+ (Smirnov et al., 2003, as Rh. kamelinii).

Rhammatophyllum kamelinii (Botsch.) Al-Shehbaz et O. Appel = Rh. erysimoides

Rorippa barbareifolia (DC.) Kitag., 1937, J. Jap. Bot. 13 (2): 137. - Camelina barbareifolia DC., 1821, Reg. Veg. Syst. Nat. 2: 517. - R. hispida suct. non (Desv.) Britt. -2.

+ Rorippa dogadovae Tzvel., 1957, Not. Syst. Herb. Inst. Bot. Acad. Sci. URSS 18: 98. - R. islandica subsp. dogadovae (Tzvel.) Jonsell, 1968, Symb. Bot. Upsal. 19: 156. - 3+, 11+ (Ebel, 2000b).

Rorippa palustris (L.) Bess., 1822, Enum. Pl. Volhyn.: 27. - Sisymbrium amphibium L. [var.] a palustre L., 1753, Sp. Pl. 2: 657. - R. islandica subsp. fernaldiana (Butters \& Abbe) Hultén, 1967, Ark. Bot., n. s. 7 (1): 61. - R. islandica auct. non (Oeder) Borbás - 1, 2, 3, 4, 5, 6, 7, 9, 10, 11, 13.

Sinapis arvensis L., 1753, Sp. Pl. 2: 663. - 4, 5+ (German, 2009), 8, 10+ (German, 2009).

+ Sisymbrium altissimum L., 1753, Sp. P1. 2: 659. - 2 (German, 2011c).

Sisymbrium brassiciforme C. A. Mey., 1831, in Ledeb., Fl. Alt. 3: 129. - 7, 14, 15+ (Smirnov et al., 2003).

Sisymbrium heteromallum C. A. Mey., 1831, in Ledeb., Fl. Alt. 3: 132. - 2, 3, 4, 6, 7, 8, 10, 11, 12, 13, 14.

Sisymbrium loeselii L., 1755, Cent. Pl. 1: 18. - 3, 4, 7+ (this study), 14.

Sisymbrium polymorphum (Murr.) Roth, 1830, Mant. Bot. 2: 946. - Brassica polymorpha Murr., 1776, Novi Comment. Gotting. 7: 35. - S. junceum (Willd.) M. Bieb., 1808, Fl. Taur.-Cauc. 2: 114. - 3, 4, 6, 7, 8, 9, 10, 14.

Sisymbrium subspinescens Bunge, 1847, Arb. Naturf. Veg. Riga 1, 2: 151. - 14.

+ Sisymbrium volgense M. Bieb. ex Fourn., 1865, Rech. Fam. Crucif.: 97. - 4+ (Smirnov et al., 2003).

Smelowskia alba (Pall.) Regel, 1861, Bull. Soc. Nat. Mosc. 34 (3): 208. - Sisymbrium album Pall., 1776, Reise 3: 293, 739. - 1, 3, 4, 6, 7, 10, 13.

Smelowskia altaica (Pobed.) Botsch., 1968, Novit. Syst. P1. Vasc. 5: 142. - Hedinia altaica Pobed., 1966, Novit. Syst. Pl. Vasc. 3: 117. - 6, 7. - Subendemic; described from Russian Altai (Ukok Plateau) with paratypes from Mongolian Altai; holotype - LE.

Smelowskia bifurcata (Ledeb.) Botsch., 1968, Novit. Syst. P1. Vasc. 5: 140. - Hutchinsia bifurcata Ledeb. 1841, Fl. Ross. 1 (1): 201. - 1.

Smelowskia calycina (Steph. ex Willd.) C. A. Mey., 1831, in Ledeb. Fl. Alt. 3: 170, s. str. - Lepidium calycinum Steph. ex Willd., 1800, Sp. Pl. 3 (1): 433. - 3, 6, 7, 14 ? 
+ Smelowskia calycina subsp. pectinata (Bunge ex Ledeb.) D. A. German, 2005, Novit. Syst. Pl. Vasc. 37: 297. - Hutschinsia pectinata Bunge ex Ledeb., 1841, Fl. Ross. 1 (1): 201. - S. pectinata (Bunge ex Ledeb.) E. Veliczk., 1976, Novit. Syst. P1. Vasc. 13: 130. - S. koeltzii auct. non (Rech. f.) Rech. f. - 3+, 7+ (Ebel, 1999, as S. pectinata), 13+ (Revushkin et al., 2001, as S. pectinata), 14+ (Ebel, 1999, as S. pectina$t a$ ). - Subendemic; described from SW Siberia (SE Altai); lectotype (German, 2005a) - LE, isolectotypes B, GOET, HAL, KW, LE, M.

Smelowskia mongolica Kom., 1911, Feddes Repert. 9: 393. - Sophiopsis mongolica (Kom.) N. Busch, 1939, Fl. URSS 8: 87. - Hedinia mongolica (Kom.) E. Veliczk., 1974, Biol. Nauki 17, 6 (126): 64. - 3. Endemic; described from Khangai phytogeographical region (valley of Sagistai); holotype - LE.

Sterigmostemum botschantzevii Grub. = Microstigma deflexum (Botschantzev, 1979, in sched. typ. (LE); Jacquemoud, 1988).

Sterigmostemum regeliorum Kamelin et D. A. German $=$ S. violaceum (Zhou et al., 2001)

\# Sterigmostemum violaceum (Botsch.) H. L. Yang, 1987, F1. Desert. R. P. Sin. 2: 64. - Oreoloma violaceum Botsch., 1980, Bot. Zhurn. 65 (3): 426. - O. sulphureum Botsch., 1980, Bot. Zhurn. 65 (3): 427.S. regeliorum Kamelin et D. A. German, 2001, Turczaninowia 4, 3: 8, non (Banks et Soland.) Bornm., 1911.7, 14. - Subendemic; described from Dzungarian Gobi phytogeographical region within Mongolia (loc. Tsagan-Tunge to the south-east of the lake Gashun-Nor); holotype - LE.

Stevenia alyssoides Adams et Fisch., 1817, Mém. Soc. Nat. Mosc. 5: 84, s. str. - 1, 3.

Stevenia alyssoides subsp. zinaidae (Malysch.) Kamelin, 1995, Bot. Zhurn. 80, 3: 71. - S. zinaidae Malysch., 1963, Not. Syst. Herb. Inst. Bot. Acad. Sci. URSS 22: 16. - 1, 3. - Subendemic; described from SE Siberia (Buryatia, E Sayan); holotype and isotype - LE.

\# Stevenia canescens (DC.) D. A. German, 2007, Key P1. Tuva Rep.: 194. - Alyssum canescens DC., 1821, Reg. Veg. Syst. Nat. 2: 322, nom. prop. cons. (German, 2013b). - Ptilotrichum canescens (DC.) C. A. Mey., 1831, in Ledeb., Fl. Alt. 3: 66. - 1, 2, 3, 4, 6, 7, 8, 9, 11, 12, 13, 15, 16.

Occurrence of $S$. canescens in eastern regions of the country requires clarification.

Stevenia cheiranthoides DC., 1821, Reg. Veg. Syst. Nat. 2: 210, s. str. - Arabis incarnata Pall. ex C. A. Mey., 1831, in Ledeb., Fl. Alt. 3: 210, nom. illeg. superfl. - Draba multiceps Kitag., 1935, Rep. First Sci. Exped. Manchoukou, sect. 4, 2: 18. - 4, 5, 9+ (Neuffer et al., 2012). - Subendemic; described from SE Siberia (Transbaikal Prov., to the west of Gazimursky Zavod); lectotype (Kamelin, 1995) - G.

Stevenia cheiranthoides subsp. incarnata (Kamelin) D. A. German, 2015, Turczaninowia 18, 2: 62. S. incarnata Kamelin, 1995, Bot. Zhurn. 80, 3: 73. - Hesperis rupestris Pall., 1776, Reise 3: 260, nom. rej. prop. (German, 2013a). - Cheiranthus fruticulosus J. Mayer, 1786, Abh. Böhm. Ges. Wiss. (Math.-Nat.), 2: 237, non L., 1753. - 1, 2, 3, 4, 6, 7, 8, 10.

\# Stevenia dahurica (Peschkova) D. A. German et Al-Shehbaz, 2010, Nordic J. Bot. 28 (6): 649. - Ptilotrichum dahuricum Peschkova, 1979, Novit. Syst. Pl. Vasc. 15: 230. - Alyssum dahuricum (Peschkova) Al-Shehbaz, 2004, Novon 14 (2): 153. - 4, 5, 8+ (Neuffer et al., 2012), 9. - Subendemic; described from SE Siberia (Transbaikal Prov., valley of Bukukun); holotype and isotype - LE.

Stevenia incarnata Kamelin $\equiv \mathrm{S}$. cheiranthoides subsp. incarnata

Stevenia sergievskajae (Krasnob.) Kamelin et Gubanov, 1986, Bull. Soc. Nat. Mosc., Ser. Biol. 91, 6: 91. - Alyssum sergievskajae Krasnob., 1975, Bot. Zhurn. 60 (5): 664. - 3. - Subendemic; described from SE Siberia (Tuva, Sangilen Mts.); holotype - NS, isotypes - LE, NS, TK.

\# Stevenia tenuifolia (Steph. ex Willd.) D. A. German, 2010, Komarovia 6 (2): 84. - Alyssum tenuifolium Steph. ex Willd., 1800, Sp. Pl. 3: 460. - Ptilotrichum tenuifolium (Steph. ex Willd.) C. A. Mey., 1831, in Ledeb., F1. Alt. 3: 67. - P. canescens subsp. tenuifolium (Steph. ex Willd.) Hanelt et Davažamc, 1965, Feddes Repert. 70, 1-3: 31. - 2, 3, 4, 5, 6, 7+ (Revushkin et al., 2001), 8, 9, 10+ (Revushkin et al., 2001), 12, 13, 14, 15. - Subendemic; described from SE Siberia (lake Baikal); lectotype - B-W.

Western limit of distribution of the species in Mongolia needs elucidation; at least partly relevant material represents well-developed plants of S. canescens described as Ptilotrichum canescens var. elongatiformis A. L. Ebel.

Strigosella africana (L.) Botsch., 1972, Bot. Zhurn. 57 (9): 1038. - Hesperis africana L., 1753, Sp. Pl. 2: 663. - Malcolmia africana (L.) W. T. Aiton, 1812, Hort. Kew., ed. 2, 4: 121. - 10, 11.

+ Strigosella brevipes (Bunge) Botsch., 1972, Bot. Zhurn. 57 (9): 1041. - Dontostemon brevipes Bunge, 1847, Arb. Naturf. Veg. Riga 1 (2): 149. - Malcolmia karelinii Lipsky, 1903, Vid. Medd. Dansk. Nat. Foren Kjøbenhavn 25: 139. - 14+ (Smirnov et al., 2003).

Subularia aquatica L., 1753, Sp. P1. 2: 642. - 3, 6. 
Syrenia macrocarpa auct. non Vass. = Erysimum kotuchovii et E. mongolicum (German, 2005b)

Taphrospermum altaicum C. A. Mey. $\equiv$ Eutrema altaicum (Al-Shehbaz, Warwick, 2005)

Tauscheria lasiocarpa Fisch. ex DC. = Isatis gymnocarpa (Al-Shehbaz, 2012)

Tetracme quadricornis (Steph. ex Willd.) Bunge, 1836, Del. Sem. Horti Dorpat.: 8. - Erysimum quadricorne Steph. ex Willd., 1800, Sp. P1. 3 (1): 514. - 7, 14.

Thellungiella salsuginea (Pall.) O. E. Schulz $\equiv$ Eutrema salsugineum (Al-Shehbaz, Warwick, 2005)

Thlaspi arvense L., 1753, Sp. P1. 2: 646. - 1, 2, 3, 4, 6, 7 13, 14+ (German, 2009).

\# Thlaspi ceratocarpum (Pall.) Murr. 1774, Novi Comment. Gotting. 5: 26. - Thlaspi ceratocarpum Pall., 1773, Reise 2: 740. - Carpoceras ceratocarpum (Pall.) N. Busch, 1913, Fl. Sibir. Orient. Extr. 1: 119. $-6,10,14$.

Thlaspi cochleariforme DC. $=$ Noccaea thlaspidioides (Meyer, 2010)

Turritis glabra L., 1753, Sp. P1. 2: 666. - 7.

\section{Unconfirmed state and regional records of Mongolian Cruciferae (Brassicaceae)}

Table 1

Species erroneously recorded from Mongolia ${ }^{1}$.

\begin{tabular}{|c|c|c|c|}
\hline Species & $\begin{array}{l}\text { Reported for } \\
\text { Mongolia by }\end{array}$ & $\begin{array}{l}\text { Excluded from } \\
\text { the flora of } \\
\text { Mongolia by }\end{array}$ & Notes \\
\hline $\begin{array}{l}\text { Arabis amplexicaulis } \\
\text { Edgew. }\end{array}$ & $\begin{array}{l}\text { Urgamal et } \\
\text { al. (2013); } \\
\text { Oyuntsetseg } \\
\text { (2014) }\end{array}$ & Present study & $\begin{array}{l}\text { First reported by Neuffer et al. (2012) as } \\
\text { "Erysimum amplexicaule Willd." (apparently } \\
\text { a misprint of the actual determination E. } \\
\text { cheiranthoides [OSBU 20693]) and later } \\
\text { misinterpreted as Arabis amplexicaulis } \\
\text { Edgew. [E E. amplexicaule (Edgew.) Kuntze]. } \\
\text { Occurrence of Himalayan A. amplexicaulis in } \\
\text { Mongolia is impossible. }\end{array}$ \\
\hline Barbarea stricta Andrz. & $\begin{array}{l}\text { Kamelin et al. } \\
(1992)\end{array}$ & German (2009) & $\begin{array}{l}\text { The record was based on short-fruited specimens } \\
\text { of } B \text {. orthoceras. }\end{array}$ \\
\hline $\begin{array}{l}\text { Erysimum perofskianum } \\
\text { Fisch. et C. A. Mey. }\end{array}$ & $\begin{array}{l}\text { Polatschek } \\
\text { (2010) }\end{array}$ & $\begin{array}{l}\text { German } \\
(2012 a)\end{array}$ & $\begin{array}{l}\text { Cited localities are situated in China and refer to } \\
\text { E. amurense Kitag. }\end{array}$ \\
\hline $\begin{array}{l}\text { Erysimum ledebourii } \\
\text { D. A. German }\end{array}$ & $\begin{array}{l}\text { Urgamal et } \\
\text { al. (2013); } \\
\text { Oyuntsetseg } \\
\text { (2014) }\end{array}$ & Present study & $\begin{array}{l}\text { The species was reported for Mongolia with } \\
\text { a reference to Olonova et al. (2013). In fact, } \\
\text { E. ledebourii is endemic to W Altai within } \\
\text { Kazakhstan and adjacent Russia (German, Ebel, } \\
\text { 2009), exactly as indicated by Olonova et al. } \\
\text { (2013, as "E. ledebourianum Serg."). A finding } \\
\text { of the species in Mongolia is improbable. }\end{array}$ \\
\hline $\begin{array}{l}\text { Erysimum } \\
\text { leucanthemum (Steph. } \\
\text { ex Willd.) B. Fedtsch. }\end{array}$ & $\begin{array}{l}\text { Polatschek } \\
(2010)\end{array}$ & $\begin{array}{l}\text { German } \\
(2012 a)\end{array}$ & Cited specimen was collected in Kazakhstan. \\
\hline $\begin{array}{l}\text { Erysimum } \\
\text { quadrangulum Desf. }\end{array}$ & $\begin{array}{l}\text { Urgamal et al. } \\
\text { (2013) }\end{array}$ & Present study & $\begin{array}{l}\text { The report was based on the misinterpretation } \\
\text { of Altaian endemic E. kotuchovii as a synonym } \\
\text { of mainly E European/N Kazakhstanian } \\
\text { E. quadrangulum (Polatschek, 2010). }\end{array}$ \\
\hline $\begin{array}{l}\text { Erysimum siliculosum } \\
\text { (M. Bieb.) DC. }\end{array}$ & $\begin{array}{l}\text { Polatschek } \\
\text { (2010) }\end{array}$ & $\begin{array}{l}\text { German } \\
(2012 a)\end{array}$ & $\begin{array}{l}\text { Mongolia was mentioned in the general } \\
\text { distribution, no specimens were cited; any } \\
\text { confirming vouchers are absent. }\end{array}$ \\
\hline
\end{tabular}

1 Unless require special comments, the records based on differences in interpretation of limits and ranks of closely related taxa are not included. These are: Arabis sagittata auct. non (Bertol.) Scop. (actually A. borealis), Brassica rapa auct. non L. (B. campestris), Braya glabella auct. non Richardson (B. siliquosa), Draba cana auct. non Rydb. (D. lanceolata), Eruca vesicaria auct. non (L.) Cav. (E. sativa), Erysimum canescens auct. non Ehrh. (E. andrzejowskianum), E. hieraciifolium auct. non L. (E. marschallianum), Hesperis matronalis auct. non L. (H. sibirica). 
Table 1 (continuation)

\begin{tabular}{|c|c|c|c|}
\hline Species & $\begin{array}{l}\text { Reported for } \\
\text { Mongolia by }\end{array}$ & $\begin{array}{l}\text { Excluded from } \\
\text { the flora of } \\
\text { Mongolia by }\end{array}$ & Notes \\
\hline $\begin{array}{l}\text { Erysimum vassilczenkoi } \\
\text { Polatschek }\end{array}$ & $\begin{array}{l}\text { Grubov (1976), } \\
\text { Gubanov et al. } \\
(1990) \text { [both } \\
\text { as Syrenia } \\
\text { macrocarpa] }\end{array}$ & $\begin{array}{l}\text { German } \\
(2005 a)\end{array}$ & $\begin{array}{l}\text { Erysimum vassilczenkoi was shown to be } \\
\text { a local endemic of NW China; Mongolian } \\
\text { specimens with such identification } \\
\text { were recognized as E. kotuchovii and } \\
\text { E. mongolicum. }\end{array}$ \\
\hline $\begin{array}{l}\text { Erysimum virgatum } \\
\text { Roth }\end{array}$ & $\begin{array}{l}\text { Polatschek } \\
\text { (2010); } \\
\text { Oyuntsetseg } \\
\text { (2014) }\end{array}$ & Present study & $\begin{array}{l}\text { The record of Oyuntsetseg (2014) is based on } \\
\text { misinterpretation of E. cheiranthoides subsp. } \\
\text { transiliense as a synonym of E. virgatum as } \\
\text { proposed by Polatschek (2010). Records by } \\
\text { Polatschek (2010) are tentatively assigned } \\
\text { herein to E. marschallianum. }\end{array}$ \\
\hline \multirow[t]{3}{*}{$\begin{array}{l}\text { Eutrema altaicum (C. A. } \\
\text { Mey.) Al-Shehbaz et S. I. } \\
\text { Warwick }\end{array}$} & $\begin{array}{l}\text { Maximowicz } \\
(1889, \text { as } \\
\text { Taphrospermum } \\
\text { altaicum })\end{array}$ & $\begin{array}{l}\text { Ebel (2000a, as } \\
\text { T. altaicum) }\end{array}$ & $\begin{array}{l}\text { The species has long been reported for } \\
\text { Mongolia based on the single record of } \\
\text { Maximowicz (1889) from vicin. of the lake } \\
\text { Khubsugul. Ebel (2000a) emphasized that } \\
\text { the record fits neither ecology (forest belt } \\
\text { instead of alpine habitats), nor distribution } \\
\text { (species is unknown to the east of W Tuva) of } \\
E \text {. altaicum, is not confirmed by any material } \\
\text { and should be considered erroneous; this is also } \\
\text { true for subsequent two reports. Theoretically, } \\
\text { occurrence of E. altaicum cannot be excluded } \\
\text { in Khobdo region but no confirming material is } \\
\text { known yet. }\end{array}$ \\
\hline & $\begin{array}{l}\text { Al-Shehbaz } \\
\text { (2000, as } T \text {. } \\
\text { altaicum) }\end{array}$ & Present study & $\begin{array}{l}\text { The single gathering reported as if from } \\
\text { Mongolia: "Mongolia: Altai, Politoff s. n. } \\
\text { (K, LE)" cannot be from this country because } \\
\text { Politow collected only within present-day } \\
\text { Russia and Kazakhstan. The cited samples } \\
\text { apparently originate from SE Altai of Russia. }\end{array}$ \\
\hline & $\begin{array}{l}\text { Neuffer et al. } \\
\text { (2003, as } T \text {. } \\
\text { altaicum) }\end{array}$ & $\begin{array}{l}\text { (German et } \\
\text { al., 2003, as } \\
\text { Hymenolobus } \\
\text { procumbens). }\end{array}$ & $\begin{array}{l}\text { Cited specimen (OSBU 10597) reported as } \\
\text { novelty for both Khobdo and Mongolian Altai } \\
\text { regions (collected in the latter) is Hornungia } \\
\text { procumbens. }\end{array}$ \\
\hline \multirow[t]{3}{*}{$\begin{array}{l}\text { Goldbachia laevigata } \\
\text { (M. Bieb.) DC. }\end{array}$} & $\begin{array}{l}\text { Hilbig, } \\
\text { Schamsran } \\
(1976)\end{array}$ & $\begin{array}{l}\text { German (2001, } \\
\text { 2009) }\end{array}$ & $\begin{array}{l}\text { The single cited specimen ([Depression of Great } \\
\text { Lakes], "Ulaan uul bei Chovd, Halbwüste", } \\
\text { HAL 56584) is G. ikonnikovii. }\end{array}$ \\
\hline & $\begin{array}{l}\text { Gubanov } \\
(1996) \text {, as new } \\
\text { for Dzungarian } \\
\text { Gobi and Valley } \\
\text { of Lakes }\end{array}$ & $\begin{array}{l}\text { German }(2001, \\
\text { 2009) }\end{array}$ & $\begin{array}{l}\text { The record from Dzungarian Gobi is based } \\
\text { on specimens of G. pendula (LE). Regarding } \\
\text { the Valley of Lakes, although no material of } \\
\text { "G. laevigata" has been found on which the } \\
\text { record from that region was based, relevant } \\
\text { information should be considered erroneous as } \\
\text { no reliable findings of the species are known } \\
\text { from Mongolia and neighboring regions of } \\
\text { China and Russia. }\end{array}$ \\
\hline & $\begin{array}{l}\text { Neuffer et al. } \\
(2003) \text {, as new } \\
\text { for Khobdo and } \\
\text { Mongolian Altai }\end{array}$ & $\begin{array}{l}\text { (German et al., } \\
\text { 2003) }\end{array}$ & $\begin{array}{l}\text { Cited specimen ([Khobdo region], near Bayan- } \\
\text { Ulgiy, OSBU 10601) reported as novelty for } \\
\text { both Khobdo and Mongolian Altai regions is } G \text {. } \\
\text { ikonnikovii. }\end{array}$ \\
\hline Isatis tinctoria $\mathrm{L}$. & Grubov (1977) & $\begin{array}{l}\text { Smirnov et al. } \\
(2003)\end{array}$ & $\begin{array}{l}\text { Cited specimen and further gatherings } \\
\text { determined as I. tinctoria were re-identified as } \\
\text { I. costata. }\end{array}$ \\
\hline
\end{tabular}


Table 1 (continuation)

\begin{tabular}{|c|c|c|c|}
\hline Species & $\begin{array}{l}\text { Reported for } \\
\text { Mongolia by }\end{array}$ & $\begin{array}{l}\text { Excluded from } \\
\text { the flora of } \\
\text { Mongolia by }\end{array}$ & Notes \\
\hline $\begin{array}{l}\text { Lepidium densiflorum } \\
\text { Schrad. }\end{array}$ & Grubov (1982) & Ebel (2001) & $\begin{array}{l}\text { Ebel (2001) demonstrated that by the end of } \\
\text { XX century, the name L. densiflorum was } \\
\text { widely misapplied to the plants of L. apetalum } \\
\text { from N and Central Asia. Indeed, all revised } \\
\text { collections of " } L \text {. densiflorum" from Mongolia } \\
\text { represent } L \text {. apetalum, in agreement with earlier } \\
\text { data of Thellung (1906). At the same time, } \\
\text { American } L \text {. densiflorum is becoming common } \\
\text { in neighboring China and Russia and will very } \\
\text { likely be introduced in Mongolian but presently } \\
\text { it is not confirmed yet. }\end{array}$ \\
\hline Lepidium latifolium $\mathrm{L}$. & $\begin{array}{l}\text { Grubov (1955, } \\
\text { 1982) }\end{array}$ & Present study & 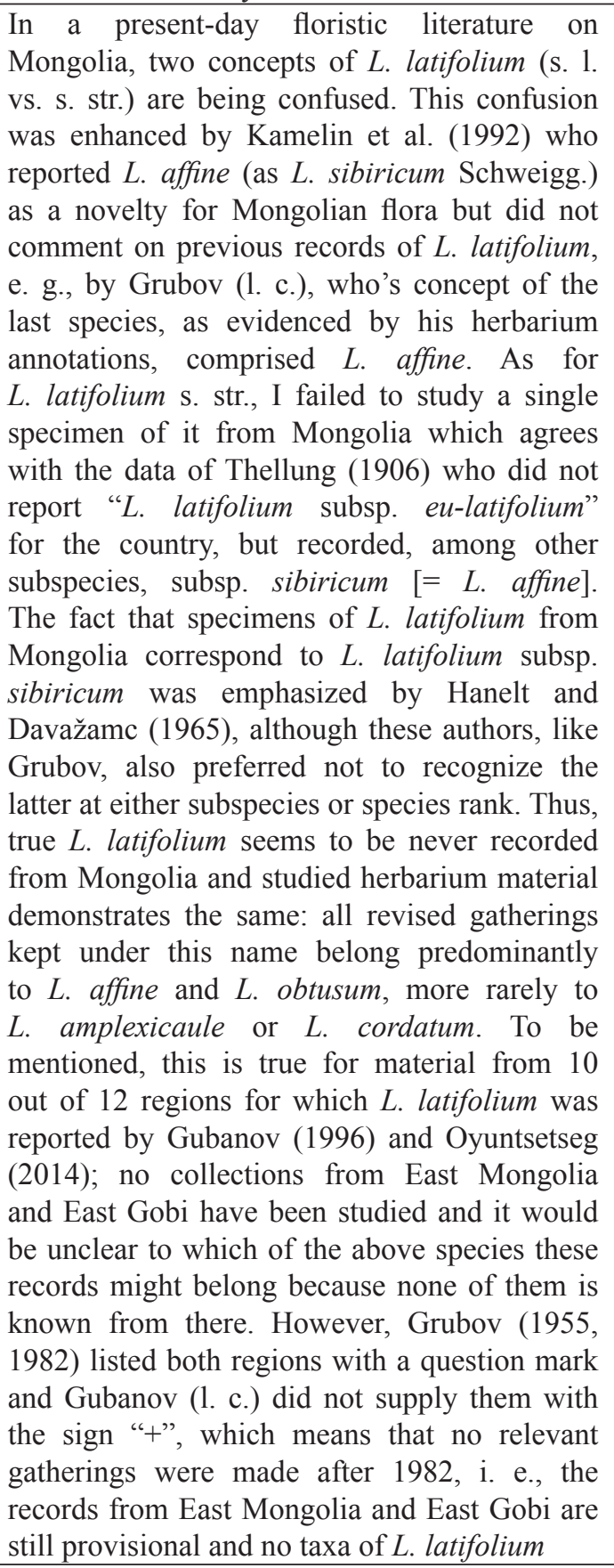 \\
\hline
\end{tabular}


Table 1 (continuation)

\begin{tabular}{|c|c|c|c|}
\hline Species & $\begin{array}{l}\text { Reported for } \\
\text { Mongolia by }\end{array}$ & $\begin{array}{l}\text { Excluded from } \\
\text { the flora of } \\
\text { Mongolia by }\end{array}$ & Notes \\
\hline & & & $\begin{array}{l}\text { affinity are yet collected from these regions. } \\
\text { In sum, the possibility of finding } L \text {. latifolium s. } \\
\text { str. in Mongolia cannot be excluded in view of } \\
\text { its ability of being weedy and introduced (conf. } \\
\text { Thellung, 1. c.), but currently its occurrence in } \\
\text { the country is not confirmed. }\end{array}$ \\
\hline Lepidium ruderale L. & $\begin{array}{l}\text { Urgamal et } \\
\text { al. (2013); } \\
\text { Oyuntsetseg } \\
(2014)\end{array}$ & Present study & $\begin{array}{l}\text { In both works the species is reported for } \\
\text { Mongolia with reference to Grubov (1982) } \\
\text { and Manibazar (2010). The latter work is the } \\
\text { Dictionary of Latin and Mongolian plants names } \\
\text { and cannot be considered a source of floristic } \\
\text { data. In Grubov (1982), L. ruderale is given as } \\
\text { a species which "could be found along the roads } \\
\text { and in settlements as a weed" but not a single } \\
\text { locality is reported. By this, Grubov corrected } \\
\text { his earlier data: in the "Conspect ..." (Grubov, } \\
\text { 1955) the species was reported for up to } 9 \\
\text { regions of the country. Renewed information } \\
\text { is in agreement with the herbarium material: } \\
\text { all gatherings of "L. ruderale" from Mongolia } \\
\text { revised by Grubov represent } L \text {. apetalum and } \\
\text { this is true for subsequent collections with such } \\
\text { determination I could check. An old report of } \\
\text { Thellung (1906: 137-138): "West-Mongolei } \\
\text { (Valé de Kéroulen, 1895-6, Chaffanjon - } \\
\text { Paris)" is noteworthy as it is based on the } \\
\text { correctly determined specimen: "Vallée de } \\
\text { Kéroulen, Mandchourie. M. Chaffanjon, } \\
\text { 1895-1896" (P 05444728; http://coldb.mnhn.fr/ } \\
\text { catalognumber/mnhn/p/p05444728). As follows } \\
\text { from the label, it was apparently collected in the } \\
\text { Chinese (Mandshurian) part of the valley of } \\
\text { Kerulen. Hence, occurrence of } L \text {. ruderale in } \\
\text { Mongolia can be considered possible but not yet } \\
\text { confirmed. }\end{array}$ \\
\hline $\begin{array}{l}\text { Lepidium songaricum } \\
\text { Schrenk }\end{array}$ & $\begin{array}{l}\text { Revushkin et al. } \\
\text { (2001); Ebel, } \\
\text { Rudaya (2002) }\end{array}$ & German (2009) & $\begin{array}{l}\text { Both reports are based on the poorly developed } \\
\text { plants of L. lacerum. }\end{array}$ \\
\hline $\begin{array}{l}\text { Neotorularia korolkowii } \\
\text { (Regel et Schmalh.) } \\
\text { Hedge et J. Léonard }\end{array}$ & $\begin{array}{l}\text { Maximowicz } \\
\text { (1880, as } \\
\text { Malcolmia } \\
\text { mongolica } \\
\text { Maxim.); } \\
\text { Grubov (1979); } \\
\text { Jäger et al. } \\
\text { (1985) [both } \\
\text { as Torularia } \\
\text { korolkowii] }\end{array}$ & German (2009) & $\begin{array}{l}\text { Among three records from Mongolia, the } \\
\text { latter is Strigosella brevipes (HAL 57361). } \\
\text { The second is not located but ecologically } \\
\text { and geographically does not correspond to } \\
\text { N. korolkowii. The first is indeed N. korolkowii } \\
\text { (lectotype of Malcolmia mongolica, LE), but, } \\
\text { along with a set of gatherings of other species by } \\
\text { Dr. Kalning from "Kobdo" in 1870, it apparently } \\
\text { originates from outside Mongolia, most likely E } \\
\text { Tian Shan (details in German, 2009). } \\
\text { Grubov (1955) questioned that Kalning } \\
\text { collected the species in Khobdo area but later } \\
\text { (Grubov, 1982) listed it without question mark. }\end{array}$ \\
\hline
\end{tabular}


Table 1 (end)

\begin{tabular}{|c|c|c|c|}
\hline Species & $\begin{array}{l}\text { Reported for } \\
\text { Mongolia by }\end{array}$ & $\begin{array}{l}\text { Excluded from } \\
\text { the flora of } \\
\text { Mongolia by }\end{array}$ & Notes \\
\hline $\begin{array}{l}\text { Neotorularia } \\
\text { maximowiczii (Botsch.) } \\
\text { Botsch. }\end{array}$ & $\begin{array}{l}\text { Grubov (1972, } \\
\text { as Torularia } \\
\text { maximowiczii } \\
\text { Botsch.) }\end{array}$ & German (2001) & $\begin{array}{l}\text { Records from Mongolia are based on specimens } \\
\text { of Braya humilis; N. maximowiczii is recently } \\
\text { synonymized with B. stigmatosa (Franch.) Al- } \\
\text { Shehbaz et D.A. German endemic to China (Al- } \\
\text { Shehbaz, German, 2014). }\end{array}$ \\
\hline $\begin{array}{l}\text { Sterigmostemum } \\
\text { regeliorum } \text { Kamelin et } \\
\text { D. A. German }\end{array}$ & $\begin{array}{l}\text { Urgamal et al. } \\
\text { (2013) }\end{array}$ & Present study & $\begin{array}{l}\text { Sterigmostemum regeliorum has been } \\
\text { proposed as a replacing name for Oreoloma } \\
\text { sulphureum Botsch. while transferring the } \\
\text { latter to Sterigmostemum in order to avoid } \\
\text { creation of a later homonym of } S \text {. sulphureum } \\
\text { (Banks. et Soland.) Bornm., } 1911 \text { (Kamelin, } \\
\text { German, 2001). Nothing new was said about } \\
\text { its distribution, i. e., it was treated endemic to } \\
\text { NW China following the original publication } \\
\text { (Botschantzev, 1980). Zhou et al. (2001) } \\
\text { found it conspecific with S. violaceum (as } \\
\text { O. violaceum), and this viewpoint is accepted } \\
\text { here. However, would } S \text {. regeliorum be treated } \\
\text { specifically distinct from } S \text {. violaceum as done } \\
\text { by Urgamal et al. (2013) and Oyuntsetseg } \\
\text { (2014), it should not be reported for Mongolia } \\
\text { as long as this yellow-flowered variety of } \\
S \text {. violaceum is only known from Chinese part } \\
\text { of Dzungaria. }\end{array}$ \\
\hline
\end{tabular}

Table 2

Species erroneously recorded from certain phytogeographical regions of Mongolia.

\begin{tabular}{|c|c|c|c|c|}
\hline Species & Region & Reported by & Excluded by & Notes \\
\hline $\begin{array}{l}\text { Aphragmus } \\
\text { involucratus }\end{array}$ & 6 & Grubov (1982) & $\begin{array}{l}\text { German } \\
(2009)\end{array}$ & $\begin{array}{l}\text { The single reported locality (Kity-Tai) is } \\
\text { situated in neighboring Russia (border area of } \\
\text { Tuva and Altai Republic). }\end{array}$ \\
\hline Arabis borealis & 7 & $\begin{array}{l}\text { Hilbig, Scham- } \\
\text { sran (1976); } \\
\text { Grubov (1982) } \\
\text { [both as } A \text {. } \\
\text { hirsuta] }\end{array}$ & $\begin{array}{l}\text { German } \\
(2009, \text { as } \\
\text { A. sagittata })\end{array}$ & $\begin{array}{l}\text { The single gathering cited by Hilbig \& } \\
\text { Schamsran (1976) was not located at HAL. } \\
\text { Other specimens from Mongolian Altai in } \\
\text { HAL, LE, and UBA ever determined as } A \text {. } \\
\text { sagittata }(\text { A. hirsuta) belong to other species. }\end{array}$ \\
\hline $\begin{array}{l}\text { Camelina } \\
\text { microcarpa }\end{array}$ & 6 & Grubov (1982) & $\begin{array}{l}\text { German } \\
(2009)\end{array}$ & $\begin{array}{l}\text { Neither specimens nor localities were cited; } \\
\text { material from the region is absent; occurrence } \\
\text { is possible but currently not confirmed. }\end{array}$ \\
\hline $\begin{array}{l}\text { Cardamine } \\
\text { bellidifolia }\end{array}$ & 13 & $\begin{array}{l}\text { Neuffer et al. } \\
(2012)\end{array}$ & $\begin{array}{l}\text { Present } \\
\text { study }\end{array}$ & $\begin{array}{l}\text { Cited specimen (OSBU 12084) is Aphragmus } \\
\text { involucratus. }\end{array}$ \\
\hline $\begin{array}{l}\text { Catolobus } \\
\text { pendulus }\end{array}$ & 8 & $\begin{array}{l}\text { Neuffer et al. } \\
(2012)\end{array}$ & $\begin{array}{l}\text { Present } \\
\text { study }\end{array}$ & $\begin{array}{l}\text { Cited locality (Hustai National park) is situated } \\
\text { in Mongol-Daurian region; occurrence in } \\
\text { Middle Khalkha is possible but currently not } \\
\text { confirmed. }\end{array}$ \\
\hline $\begin{array}{l}\text { Chorispora } \\
\text { bungeana }\end{array}$ & $\begin{array}{c}6,13 \\
14\end{array}$ & $\begin{array}{l}\text { Oyuntsetseg } \\
(2014)\end{array}$ & $\begin{array}{l}\text { Present } \\
\text { study }\end{array}$ & $\begin{array}{l}\text { The species was long reported for Mongolia } \\
\text { from the single locality (Kyzyl-Gaya [= } \\
\text { Kairty-Daba] pass) (e. g., Grubov, 1955, 1982) } \\
\text { in Mongolian Altai at the border with China. } \\
\text { The record is based on the gathering of V.V. } \\
\text { Sapozhnikov of } 15 \text { Jul. } 1908 \text { (TK); other } \\
\text { material on Ch. bungeana }\end{array}$ \\
\hline
\end{tabular}


Table 2 (continuation)

\begin{tabular}{|c|c|c|c|c|}
\hline Species & Region & Reported by & Excluded by & Notes \\
\hline & & & & $\begin{array}{l}\text { from Mongolia is unknown to me. Probably } \\
\text { the species was confused with Ch. sibirica } \\
\text { which is surprisingly not reported for Khobdo } \\
\text { and Dzungarian Gobi (Oyuntsetseg, 2014) } \\
\text { although it is common in these regions and } \\
\text { recorded by previous authors. }\end{array}$ \\
\hline $\begin{array}{l}\text { Conringia } \\
\text { planisiliqua }\end{array}$ & 6 & Grubov (1982) & $\begin{array}{l}\text { German } \\
(2009)\end{array}$ & $\begin{array}{l}\text { No specimens or localities were cited; material } \\
\text { from Khobdo region is absent, ecological and } \\
\text { geographical evidences are not in favour of } \\
\text { occurrence of the species in the region. }\end{array}$ \\
\hline $\begin{array}{l}\text { Dendroarabis } \\
\text { fruticulosa }\end{array}$ & 6 & $\begin{array}{l}\text { Grubov (1982, } \\
\text { as Arabis } \\
\text { fruticulosa) }\end{array}$ & $\begin{array}{l}\text { German } \\
(2009 \text {, as } A \text {. } \\
\text { fruticulosa) }\end{array}$ & $\begin{array}{l}\text { Situation is the same as with Conringia } \\
\text { planisiliqua. }\end{array}$ \\
\hline \multirow[t]{3}{*}{$\begin{array}{l}\text { Dontostemon } \\
\text { crassifolius }\end{array}$} & 11 & $\begin{array}{l}\text { Gubanov } \\
\text { (1996) }\end{array}$ & $\begin{array}{l}\text { German } \\
(2003)\end{array}$ & $\begin{array}{l}\text { Revised material from Valley of Lakes } \\
\text { (MW) belongs to D. senilis; occurrence of } \\
D . \text { crassifolius in the region is rather possible } \\
\text { but currently not confirmed. }\end{array}$ \\
\hline & 14 & $\begin{array}{l}\text { Jäger et al. } \\
(1985)\end{array}$ & $\begin{array}{l}\text { German } \\
(2009)\end{array}$ & $\begin{array}{l}\text { The single record was based on misidentified } \\
\text { specimen of D. elegans (E. Jäger E } 313 \text {, HAL). }\end{array}$ \\
\hline & 15 & $\begin{array}{l}\text { Gubanov } \\
(1996)\end{array}$ & $\begin{array}{l}\text { German } \\
(2003)\end{array}$ & $\begin{array}{l}\text { The single record was based on misidentified } \\
\text { specimen of } D \text {. senilis (NW of Atas-Bogdo, } \\
\text { G. N. Ogureyeva s. n., MW). A finding of } \\
D . \quad \text { crassifolius in both Dzungarian and } \\
\text { Transaltai Gobi is unlikely. }\end{array}$ \\
\hline \multirow[t]{2}{*}{$\begin{array}{l}\text { Dontostemon } \\
\text { elegans }\end{array}$} & 6 & Grubov (1982) & \multirow{2}{*}{$\begin{array}{l}\text { German } \\
(2003) ; \\
\text { German et } \\
\text { al. (2003) }\end{array}$} & $\begin{array}{l}\text { All collections from Khobdo region and } \\
\text { Depression of Great Lakes (as well as } \\
\text { from adjacent part of Mongolian Altai) }\end{array}$ \\
\hline & 10 & $\begin{array}{l}\text { Gubanov } \\
(1996)\end{array}$ & & $\begin{array}{l}\text { ever determined as D. elegans and } \\
\text { D. crassifolius (exceptforfew specimens of true } \\
\text { D. crassifolius from Depression of Great } \\
\text { Lakes) were re-identified as "untypical } \\
\text { D. senilis" and subsequently recognized as } \\
\text { D. senilis subsp. gubanovii (German, 2007) } \\
\text { currently accepted as D. gubanovii. }\end{array}$ \\
\hline \multirow[t]{2}{*}{$\begin{array}{l}\text { Dontostemon } \\
\text { perennis }\end{array}$} & 5 & $\begin{array}{l}\text { Gubanov } \\
(1996) ; \\
\text { Gubanov et al. } \\
(1996)\end{array}$ & $\begin{array}{l}\text { German } \\
(2009)\end{array}$ & $\begin{array}{l}\text { The record was based on the collection of } \\
\text { E. Ganbold et al., } 1146 \text { (MW) re-identified } \\
\text { by A. L. Ebel as D. dentatus (Gubanov, } \\
\text { 1999). No other specimens determined as } \\
D \text {. perennis from Great Khingan are available; } \\
\text { its occurrence in the region and eastern part of } \\
\text { Mongolia as a whole (see next comment) is not } \\
\text { expected. }\end{array}$ \\
\hline & 8,12 & Grubov (1955) & $\begin{array}{l}\text { Present } \\
\text { study }\end{array}$ & $\begin{array}{l}\text { All revised collections from Middle Khalkha } \\
\text { and East Gobi ever determined as D. perennis } \\
\text { (mainly LE) were re-identified, predominantly } \\
\text { as D. integrifolius or D. senilis, by V. F. } \\
\text { Golubkova (in 1950), Ch. Sanchir (in 1977), } \\
\text { V. I. Grubov (in 1970-1980ths) and finally } \\
\text { by the present author (since 2000). Thus, } \\
\text { occurrence of D. perennis the eastern part of } \\
\text { Mongolia does not get support. The species } \\
\text { is apparently restricted to the western part of } \\
\text { the country and even there it is not as widely } \\
\text { distributed as previously thought (see the next } \\
\text { comment). }\end{array}$ \\
\hline
\end{tabular}


Table 2 (continuation)

\begin{tabular}{|c|c|c|c|c|}
\hline Species & Region & Reported by & Excluded by & Notes \\
\hline & 15 & Grubov (1982) & $\begin{array}{l}\text { Present } \\
\text { study }\end{array}$ & $\begin{array}{l}\text { There are only two specimens (Dayan Uul, } \\
5 \text { VII 1973, N.S Golubkova \& U. Tsogt } 186 \\
\text { and Khurgu Uul, } 15 \text { VII 1973, E.A. Isachenko } \\
\& \text { E. I. Rachkovskaya } 2668 \text {, both LE) from } \\
\text { Transaltai Gobi determined as D. perennis (a } \\
\text { very similar specimen, } 10 \mathrm{~km} \text { to the south of } \\
\text { Noen, V. I. Grubov et al. } 1983,8 \text { IX } 1979 \text { [LE], } \\
\text { was determined by Grubov as "D. senilis?"). } \\
\text { Habitually they fit D. senilis but in the } \\
\text { indumentum partly composed of rather short, } \\
\text { patent to subappressed trichomes, they remind } \\
D \text {. perennis. Based on to the habit, fruits, and } \\
\text { presence of long twisted trichomes they are } \\
\text { preliminarily treated as untypical specimens } \\
\text { of D. senilis and their status needs further } \\
\text { elucidation while presence of } D \text {. perennis in } \\
\text { Transaltai Gobi of Mongolia at the moment } \\
\text { cannot be confirmed. }\end{array}$ \\
\hline $\begin{array}{l}\text { Dontostemon } \\
\text { pinnatifidus }\end{array}$ & 3 & $\begin{array}{l}\text { Neuffer et al. } \\
(2012 \text {, as } D . \\
\text { pectinatus and } \\
\text { D. pinnatifidus })\end{array}$ & $\begin{array}{l}\text { Present } \\
\text { study }\end{array}$ & $\begin{array}{l}\text { The record was based on three specimens } \\
\text { (OSBU 12263, 12268, 12279) collected in } \\
\text { two localities with the same coordinates } \\
\text { corresponding to NW part of Middle Khalkha. } \\
\text { Occurrence of the species in Khangai is } \\
\text { possible but cannot be considered confirmed } \\
\text { yet. }\end{array}$ \\
\hline $\begin{array}{l}\text { Dontostemon } \\
\text { senilis }\end{array}$ & 8 & $\begin{array}{l}\text { Neuffer et al. } \\
(2012)\end{array}$ & $\begin{array}{l}\text { Present } \\
\text { study }\end{array}$ & $\begin{array}{l}\text { The record was based on two specimens } \\
\text { (OSBU } 11810 \text { and 11812) both collected near } \\
\text { the ruins of Saikhan-Ovoo (bank of Ongi-gol). } \\
\text { This locality is situated at the very east of } \\
\text { the Valley of Lakes (border with East Gobi). } \\
\text { Occurrence of D. senilis in southern part of } \\
\text { Middle Khalkha is possible but currently not } \\
\text { confirmed. }\end{array}$ \\
\hline Draba altaica & 10 & $\begin{array}{l}\text { Oyuntsetseg } \\
(2014)\end{array}$ & $\begin{array}{l}\text { Present } \\
\text { study }\end{array}$ & $\begin{array}{l}\text { The record was given with a reference to } \\
\text { German (2009); however, in the latter work } \\
\text { D. altaica is added to the flora of Gobi-Altai } \\
\text { which is not mentioned in Oyuntsetseg ( } 2014) \text {. } \\
\text { Apparently, " } 13+\text { " was misprinted as " } 10+\text { " in } \\
\text { that work. }\end{array}$ \\
\hline Draba baicalensis & 10 & $\begin{array}{l}\text { Oyuntsetseg } \\
(2014 \text {, as } D . \\
\text { czuensis) }\end{array}$ & $\begin{array}{l}\text { Present } \\
\text { study }\end{array}$ & $\begin{array}{l}\text { The single mentioned gathering (Naranbulag, } \\
\text { Uvs aimag) was collected in the north-westmost } \\
\text { part of Khangai (Khan Khukhii range, mt. } \\
\text { Khurmiin-Ovoo) and not in Depression of } \\
\text { Great Lakes as explicitly reported by Ebel in a } \\
\text { referred work (Ebel, 2002, as D. czuensis) and } \\
\text { previously by Gubanov and Kamelin (1991) } \\
\text { while reporting the species as a novelty for } \\
\text { Khangai. }\end{array}$ \\
\hline Draba mongolica & 6,7 & Grubov (1955) & Ebel (2002) & $\begin{array}{l}\text { Available material on "D. mongolica" from W } \\
\text { Mongolia was assigned to other Draba species. } \\
\text { This is also true for subsequent records (e. g., } \\
\text { Neuffer et al., 2003). }\end{array}$ \\
\hline Draba ochroleuca & 13 & Grubov (1982) & $\begin{array}{l}\text { German } \\
(2009)\end{array}$ & $\begin{array}{l}\text { Relevant specimens were re-determined as } \\
\text { subglabrous form of } D \text {. oreades. }\end{array}$ \\
\hline
\end{tabular}


Table 2 (continuation)

\begin{tabular}{|c|c|c|c|c|}
\hline Species & Region & Reported by & Excluded by & Notes \\
\hline Draba pygmaea & 6 & $\begin{array}{l}\text { Gubanov } \\
(1996)\end{array}$ & $\begin{array}{l}\text { Smirnov et } \\
\text { al. }(2003)\end{array}$ & $\begin{array}{l}\text { The specimen on which the record of } \\
D \text {. pygmaea from Khobdo region and } \mathrm{W} \\
\text { Mongolia as a whole was based is a mixture of } \\
\text { D. turczaninowii and D. altaica. }\end{array}$ \\
\hline $\begin{array}{l}\text { Erysimum } \\
\text { andrzejowskianum }\end{array}$ & $\begin{array}{c}2,3,4 \\
8,9 \\
10,13\end{array}$ & $\begin{array}{l}\text { Grubov (1955, } \\
\text { as E. diffusum) }\end{array}$ & $\begin{array}{l}\text { Present } \\
\text { study }\end{array}$ & $\begin{array}{l}\text { Since the checklist of Grubov (1955), } \\
E \text {. andrzejowskianum is reported for eight } \\
\text { regions of the country (those listed here plus } \\
\text { Mongolian Altai). Based on revised material, } \\
\text { presence of the species only in the latter region } \\
\text { can be confirmed while other collections } \\
\text { represent E. flavum s. 1. (HAL, LE). Despite } \\
\text { most of old material of "E. canescens" or } \\
\text { "E. diffusum" in LE from relevant regions } \\
\text { has been correctly re-identified by Grubov in } \\
1976 \text {, the same (wide) distribution was still } \\
\text { given in the "Key ..." (Grubov, 1982) and } \\
\text { reported in subsequent Mongolian treatments } \\
\text { (Gubanov, 1996; Oyuntsetseg, 2014, both as } \\
E \text {. canescens). To be noted, the single locality } \\
\text { in Mongolian Altai ("north-west: upper reaches } \\
\text { of Tsagan-gol") given in Grubov (1955), is } \\
\text { based on the specimen "Slope near the river } \\
\text { Dzurhe, one of the peaks of Tsagan-gol. Light } \\
\text { larch woods. 30 VII [18]98. № 76. [D.A. \& E.N. } \\
\text { Klementz]" (LE) which originates from one of } \\
\text { the tributaries of Urungu in neighboring China } \\
\text { and not from the tributary of Khobdo-gol. This } \\
\text { conclusion is in congruence with Klementz's } \\
\text { route of } 1898 \text { (Grubov, 1955: 14). However, } \\
\text { occurrence of the species in Mongolia is } \\
\text { confirmed based on recent collections from } \\
\text { Dzungarian slope of Mongolain Altai (valleys } \\
\text { of Bulgan-gol, Bodonchijn-gol - ALTB). }\end{array}$ \\
\hline $\begin{array}{l}\text { Erysimum flavum } \\
\text { s. str. }\end{array}$ & $6,7,10$ & Grubov (1982) & $\begin{array}{l}\text { Ebel } \\
(2000 a) ; \\
\text { German, } \\
\text { Oyuntsetseg } \\
(2008)\end{array}$ & $\begin{array}{l}\text { The species is represented in relevant regions } \\
\text { by subsp. altaicum. }\end{array}$ \\
\hline $\begin{array}{l}\text { Eutrema } \\
\text { salsugineum }\end{array}$ & 6 & $\begin{array}{l}\text { Neuffer et } \\
\text { al. (2003, as } \\
\text { Thellungiella } \\
\text { salsuginea) }\end{array}$ & $\begin{array}{l}\text { (German et } \\
\text { al., 2003, } \\
\text { as Noccaea } \\
\text { coch- } \\
\text { leariformis) }\end{array}$ & $\begin{array}{l}\text { Cited specimen (OSBU 10379) was re- } \\
\text { identified as Noccaea thlaspidioides. }\end{array}$ \\
\hline $\begin{array}{l}\text { Goldbachia } \\
\text { ikonnikovii }\end{array}$ & 9 & $\begin{array}{l}\text { Gubanov } \\
(1996)\end{array}$ & $\begin{array}{l}\text { German et } \\
\text { al. (2003) }\end{array}$ & $\begin{array}{l}\text { Occasional record from East Mongolia } \\
\text { actually refers to Khovd region; it is based on } \\
\text { a misprint ( } 9 \text { instead of } 6) \text {. }\end{array}$ \\
\hline \multirow[t]{2}{*}{ Isatis oblongata } & 6 & $\begin{array}{l}\text { Hilbig, } \\
\text { Schamsran } \\
(1980,1985)\end{array}$ & $\begin{array}{l}\text { German } \\
(2009)\end{array}$ & $\begin{array}{l}\text { The specimen (HAL 56302) first reported as "I. } \\
\text { costata vel I. oblongata" and later referred to } \\
\text { the latter species was re-identified as I. costata. }\end{array}$ \\
\hline & 7 & $\begin{array}{l}\text { Gubanov } \\
\text { (1996) }\end{array}$ & $\begin{array}{l}\text { German } \\
(2009)\end{array}$ & $\begin{array}{l}\text { The single specimen identified as I. oblongata } \\
\text { from Mongolian Altai (Gubanov Nr. 8972, } \\
\text { MW) is I. costata. }\end{array}$ \\
\hline $\begin{array}{l}\text { Lepidium } \\
\text { cartilagineum }\end{array}$ & 6 & $\begin{array}{l}\text { Neuffer et al. } \\
\text { (2003, as } L \text {. } \\
\text { crassifolium) }\end{array}$ & $\begin{array}{l}\text { German et } \\
\text { al. (2003) }\end{array}$ & $\begin{array}{l}\text { Cited specimen (OSBU 10467) was re- } \\
\text { identified as L. cordatum. }\end{array}$ \\
\hline
\end{tabular}


Table 2 (continuation)

\begin{tabular}{|c|c|c|c|c|}
\hline Species & Region & Reported by & Excluded by & Notes \\
\hline $\begin{array}{l}\text { Microstigma } \\
\text { brachycarpum }\end{array}$ & 6,7 & $\begin{array}{l}\text { Neuffer et al. } \\
\text { (2003, as } M \text {. } \\
\text { junatovii) }\end{array}$ & $\begin{array}{l}\text { Present } \\
\text { study }\end{array}$ & $\begin{array}{l}\text { The species was reported for both Khobdo } \\
\text { region and Mongolian Altai based on the } \\
\text { single gathering. Unfortunately, relevant } \\
\text { specimen (OSBU 10809) seems to be } \\
\text { misplaced and could not be revised. However, } \\
\text { a possibility of occurrence of the species } \\
\text { in the area is definitely excluded based on } \\
\text { severe discrepancy between the ecology and } \\
\text { geography of } M \text {. brachycarpum (desert plant } \\
\text { known from the very south of the country) and } \\
\text { collection data (alpine habitat at } 2800 \text { m a. s. } 1 \text {. } \\
\text { at the ultimate north-west of Mongolia [Tavan } \\
\text { Bogd mountain knot]). }\end{array}$ \\
\hline $\begin{array}{l}\text { Microstigma } \\
\text { deflexum }\end{array}$ & 15 & $\begin{array}{l}\text { Gubanov et al. } \\
\text { (1987) }\end{array}$ & $\begin{array}{l}\text { German } \\
(2009 \text {, as } M \text {. } \\
\text { junatovii) }\end{array}$ & $\begin{array}{l}\text { The single cited specimen (MW) represents } \\
\text { M. brachycarpum. }\end{array}$ \\
\hline $\begin{array}{l}\text { Noccaea } \\
\text { thlaspidioides }\end{array}$ & 11 & $\begin{array}{l}\text { Neuffer et } \\
\text { al. (2012, } \\
\text { as Thlaspi } \\
\text { cochleariforme) }\end{array}$ & $\begin{array}{l}\text { Present } \\
\text { study }\end{array}$ & $\begin{array}{l}\text { This record was based on the single specimen } \\
\text { (OSBU 12247) actually collected in southern } \\
\text { Khangai. }\end{array}$ \\
\hline Rorippa palustris & 8 & $\begin{array}{l}\text { Neuffer et al. } \\
\text { (2012, as } R \text {. } \\
\text { islandica subsp. } \\
\text { fernaldiana) }\end{array}$ & $\begin{array}{l}\text { Present } \\
\text { study }\end{array}$ & $\begin{array}{l}\text { The record was based on the specimen (OSBU } \\
11794 \text { ) collected, along with Dontostemon } \\
\text { senilis, near the ruins of Saikhan-Ovoo (bank } \\
\text { of Ongi-gol), i. e., in the Valley of Lakes. } \\
\text { Occurrence of } R \text {. palustris in Middle Khalkha } \\
\text { is possible but currently not confirmed. }\end{array}$ \\
\hline \multirow{3}{*}{$\begin{array}{l}\text { Smelowskia } \\
\text { calycina }\end{array}$} & 1 & Grubov (1955) & \multirow[t]{3}{*}{ Ebel (1999) } & \multirow[b]{3}{*}{$\begin{array}{l}\text { According to Ebel (1999), S. calycina } \\
\text { [s. str.] occurs within Mongolia only in } \\
\text { Khangai and Mongolian Altai; apparently, } \\
\text { the latter is meant as a mountain range rather } \\
\text { than phytogeographical region and thus } \\
\text { includes Khobdo region. Hence, records } \\
\text { from Khubsugul region should be assigned } \\
\text { to } S \text {. bifurcata and those from Gobi-Altai } \\
\text { and Dzungarian Gobi to } S \text {. calycina subsp. } \\
\text { pectinata. However, some specimens from } \\
\text { Baitag Bogd approach } S \text {. calycina s. str. and } \\
\text { presence of the taxon in Dzungarian Gobi } \\
\text { needs further elucidation. }\end{array}$} \\
\hline & 13 & Grubov (1982) & & \\
\hline & 14 & $\begin{array}{l}\text { Gubanov } \\
(1996)\end{array}$ & & \\
\hline \multirow[t]{2}{*}{$\begin{array}{l}\text { Smelowskia } \\
\text { mongolica }\end{array}$} & 6 & $\begin{array}{l}\text { Neuffer et al. } \\
(2003)\end{array}$ & $\begin{array}{l}\text { German et } \\
\text { al. }(2003)\end{array}$ & $\begin{array}{l}\text { Both cited specimens (OSBU 10301, 10413) } \\
\text { were re-identifies ad S. altaica. }\end{array}$ \\
\hline & 7 & $\begin{array}{l}\text { Grubov (1955, } \\
\text { as Sophiopsis } \\
\text { mongolica } \\
\text { (Kom.) N. } \\
\text { Busch) }\end{array}$ & $\begin{array}{l}\text { Ebel (1999, } \\
\text { 2000a, as } \\
\text { Hedinia } \\
\text { mongolica) }\end{array}$ & $\begin{array}{l}\text { The specimens of S. mongolica from } \\
\text { Mongolian Altai were assigned to } S \text {. altaica } \\
\text { (including paratypes of the latter species from } \\
\text { Tsagan-gol and Tal-Nuur). Some subsequent } \\
\text { records (e. g., Neuffer et al., 2003) from this } \\
\text { region are also based on plants of S. altaica } \\
\text { (German et al., 2003). }\end{array}$ \\
\hline $\begin{array}{l}\text { Sterigmostemum } \\
\text { violaceum }\end{array}$ & 6 & $\begin{array}{l}\text { Grubov }(1982, \\
\text { as Oreoloma } \\
\text { violaceum })\end{array}$ & $\begin{array}{l}\text { Present } \\
\text { study }\end{array}$ & $\begin{array}{l}\text { Like in case of Conringia planisiliqua and } \\
\text { Dendroarabis fruticulosa, no specimens } \\
\text { or localities were cited and no confirming } \\
\text { material from Khobdo region is available; } \\
\text { occurrence of the species in that area is very } \\
\text { unlikely. }\end{array}$ \\
\hline
\end{tabular}


Table 2 (end)

\begin{tabular}{|c|c|c|c|c|}
\hline Species & Region & Reported by & Excluded by & Notes \\
\hline \multirow[t]{2}{*}{$\begin{array}{l}\text { Stevenia } \\
\text { cheiranthoides }\end{array}$} & 6 & $\begin{array}{l}\text { Neuffer et al. } \\
\text { (2003, as Draba } \\
\text { multiceps) }\end{array}$ & $\begin{array}{l}\text { Present } \\
\text { study }\end{array}$ & $\begin{array}{l}\text { The record is based on the misidentified } \\
\text { specimen of Draba lanceolata (OSBU 10262). }\end{array}$ \\
\hline & 7 & $\begin{array}{l}\text { Neuffer et al. } \\
(2003)\end{array}$ & $\begin{array}{l}\text { Present } \\
\text { study }\end{array}$ & $\begin{array}{l}\text { The record is based on the misidentified } \\
\text { specimen of Crucihimalaya mollissima } \\
\text { (OSBU 10589: } \\
\text { http://greif.uni-greifswald.de/floragreif/ } \\
\text { wp-content/uploads/scan_OSBU/template. } \\
\text { php?i=arab-moll-OSBU-10589). }\end{array}$ \\
\hline \multirow[t]{2}{*}{ Stevenia dahurica } & 2 & $\begin{array}{l}\text { Neuffer et } \\
\text { al. (2012), as } \\
\text { Ptilotrichum } \\
\text { dahuricum }\end{array}$ & $\begin{array}{l}\text { Present } \\
\text { study }\end{array}$ & $\begin{array}{l}\text { The record is based on two specimens (OSBU } \\
\text { 20346, 20372) attributed to Khentei but } \\
\text { collected in Mongol-Daurian region. }\end{array}$ \\
\hline & 12 & $\begin{array}{l}\text { Grubov et al. } \\
(1990) \text {, as } P \text {. } \\
\text { dahuricum }\end{array}$ & $\begin{array}{l}\text { German } \\
(2009 \text {, as } P \text {. } \\
\text { dahuricum })\end{array}$ & $\begin{array}{l}\text { Identification of relevant specimens is } \\
\text { problematic but they definitely do not belong } \\
\text { to } S \text {. dahurica. Further studies are needed } \\
\text { to either recognize them as a new species } \\
\text { ("Prilotrichum gobicum Kamelin et D. A. } \\
\text { German", ined.) or assign to } S \text {. canescens. }\end{array}$ \\
\hline
\end{tabular}

III. Geographical notes: new country record, regional records, and remarks on distribution of certain species

\section{Novelty for the flora of Mongolia}

Matthiola superba Conti - Dzungarian Gobi: "Aimak-Chovd, Somon Bulgan, Biljut-berge nördl. d. Grenzpunktes Jarantaj a. Bulgan, Berghänge. Meereshöhe 1200 m. 4. 7. 1964. Nr. 3481. Leg. S. Danert, C. Davažamc, P. Hanelt et Č. Sančir" (GAT 9233, sub nom. Sisymbrium subspinescens).

This is the first record of Matthiola W. T. Aiton from Mongolia. Matthiola superba is the most eastern representative of the genus reaching the western part of SE Siberia, E Kazakhstan and NW Xinjiang.

According to the current knowledge, distribution of the species is rather fragmented in this area and the nearest localities known from northern foothills of Saur within Kazakhstan (Krylov, 1931, as M. odoratissima (M. Bieb.) R. Br.), vicinities of Bortala and Urumqi (German, Chen, 2009) in China are quite distant (300 $\mathrm{km}$ and more) from each other and from the Mongolian site.

A long-known peculiarity of the specimens from the eastern part of its distribution area is rather considerable reduction of indumentum (Chernyakovskaya, 1939), especially in fruit. The plant from Bilyut-Ula, similarly to the geographically closest specimen from Urumqi (Anonymous 3175, XJU: German, Chen, 2009) reveals the ultimate case ha- ving trichomes almost exclusively restricted to the stem base; therefore, its initial identification is not too surprising.

The species definitely requires protection at the state level in Mongolia. Inclusion into the next edition of the national Red Data book and monitoring of the single known Mongolian population, if still persisting, could be the first steps in this direction.

\section{Regional records}

Barbarea orthoceras Ledeb. - Khentei: "Mongolei, Ulan-Bator, Bogd-uul, feuchter Waldweg. W. Hilbig. 2. 7. 1986" (HAL 129453, sub nom. B. arcuata; http://greif.uni-greifswald.de/floragreif/wp-content/uploads/scan_GFW-14/template. php?i=Barb-arcu-HAL-129453)

Capsella orientalis Klok. - Khangai: "Khangai. Stony slopes near the spring Khatsinlin-bulak. 4 VIII 1926. No. 406. N. Pavlov" (MW); "Khangai. Zavkhan aimag. Valley of Sherigiin-gol in $5 \mathrm{~km}$ downstream from Thagan-Khairkhan. 3 VIII 1982. No. 5982. I. A. Gubanov" (MW); "Uvs aimag. Khyargas sum. Khan-Khukhii range, western slope of mt. Tsagan-Khairkhan-Uul, ca. $2150 \mathrm{~m}$ a. s. 1. Upper reaches of Khiiviin-gol, $25 \mathrm{~km} \mathrm{SW}$ of Khyargas. 5 IX 1984. No. 9252. I. A. Gubanov" (MW). Depression of Great Lakes: "Uvs Nuur depression. 14 km SE of Ulangom. Valley of Khototyn-Khev. 6 VII 1988. No. 922. I. A. Gubanov, R. V. Kamelin, A.L. 
Budantsev" (MW); "Kobdo (Khovd): Weiderasen ca. $23 \mathrm{~km}$ nordwestl. Tschandmani (Urgdol), am Fuß der Steilstufe des Jargalant-Gebirges (Dshargalant), an der Mündung eines großen klammartigen Tales, ca. 2600 m s. m. Leg. K.-H. Günther et M. Schnittler am 04. 09. 2007" (Herb. K.-H. Günther; $\mathrm{http}$ //greif.uni-greifswald.de/floragreif/wp-content/ uploads/scan_GFW-13/template.php?i=caps-bursKF-27950). All specimens - sub nom. C. bursapastoris.

Catolobus pendulus (L.) Al-Shehbaz - Depression of Great Lakes: "Kobdo [Khovd] city. Weed in the garden. 11 VII 1898. № 430. E. Klementz" (LE).

Lepidium affine Ledeb. - Middle Khalkha: "Aimak Chentij, Somon Bajan chutag, Steppen-ebene ca. $12 \mathrm{~km}$ südöstlich von Öndörchaan am Wege nach Burencogt. 31. 7. 1987. Nr. 26. Leg. K. Pistrick, Č. Sančir et G. Cerenbalžid" (GAT 7992, sub nom. L. latifolium).

Lepidium apetalum Willd. - Khovd: "Ad fontes fl. Harkira ... 25 Juli 1879. G. N. Potanin" (LE, det. A. Thellung); "Bayan-Ulgiy aimag, $18 \mathrm{~km}$ to the east of Bayan-Ulgiy. 13 VII 1979. № 73. U. Beket, Kh. Buyan-Orshikh, I. Yu. Sumerina" (LE, sub nom. L. densiflorum); "Bayan-Ulgiy, 25 VII 2001, R.V. Kamelin et al." (ALTB), etc.

Sisymbrium loeselii L. - Mongolian Altai: "Lower reaches of Sagsai-gol, 25 VII 2001. R.V. Kamelin et al." (ALTB).

\section{Other floristic notes}

Clausia aprica (Steph. ex Willd.) Korn.-Tr. - the species is not reported for Gobi-Altai by Gubanov (1996) and Oyuntsetseg (2014). Probably it was occasionally omitted by the former author because it is included in both Grubov's $(1955,1982)$ treatments and those records are supported by abundant correctly determined material from different parts of the region. In particular, gatherings from Baga Bogd, Ikh Bogd, Dund-Saikhan, Dzun-Saikhan, and Bayan-Bogdo-Nuruu are available at LE.

Pachyneurum grandiflorum (C. A. Mey.) Bunge - The species is recorded here from Depression of Great Lakes based on the gathering "Kobdo (Khovd): lückiger Rasen an einem nordexponierten Steilhang ca. $23 \mathrm{~km}$ nordwestl. Tschandmani
(Urgdol), im untersten Bereich der Steilstufe des Jargalant-Gebirges (Dshargalant), an der Mündung eines großen klammartigen Tales, ca. 2600 m s. m. Leg. K.-H. Günther et M. Schnittler am 05. 09. 2007" (Private herbarium of K.-F. Günther) correctly identified by collectors and available at http://greif.uni-greifswald.de/floragreif/?flora search=Record\&record_id $=29803$ and http:// greif.uni-greifswald.de/floragreif/wp-content/uploads/scan_GFW-13/template.php?i=pach-granKF-27971).

Rorippa dogadovae Tzvel. - The species was first recorded from Mongolia by Ebel (2000b) based on two gatherings, one from Khangai and another from Valley of Lakes. The latter specimen ("Middle Gobi aimak, Delger-Khangai sum, loc. KhutuKhid at Ongijn-gol, flood plain. 22 V 1947. Grubov et Kalinina" - LE) was said to be "bad-quality and not completely reliable" one and indeed, both plants constituting this gathering might well be just small samples of $R$. palustris. Here occurence of $R$. dogadovae in the Valley of Lakes is confirmed based on the gathering "Mongolei, $45^{\circ} 05^{\prime} 39^{\prime} \mathrm{N}$, $100^{\circ} 48^{\prime} 47^{\prime}$ 'E, ca. $1200 \mathrm{~m}$, südlich des Orrog Nuur, Glycirrhiza-Standort Flussbett, versalzter Boden. 25. 08. 2001. H. Hurka" (OSBU 12156, upper right and lower left plants; other two plants are R. palustris). The specimen was cited under the name $R$. islandica and attributed to Gobi-Altai region (Neuffer et al., 2012). In fact, it was collected at the border of the two regions, and based on the habitat and elevation it definitely sould be assigned to the Valley of Lakes.

Sisymbrium brassiciforme C. A. Mey. - [Mongolian Altai]: "Becken der Großen Seen, Khovd-sum in Khovd-Aimag, Barun Sala-Tal, Bergsteppe. Höche 2200-2500 m, trockene Bergsteppe. 11. 06. 2002. Leg. Anne Zemmrich" (GFW 45515, sub nom. S. heteromallum; http://greif.uni-greifswald.de/floragreif/?flora_search=Record\&record $\mathrm{id}=45545$ ). According to the phytogeographic region indicated on the label, this would be the first record of S. brassiciforme from Depression of Great Lakes; however, the valley of Barun-gol at the mentioned elevation belongs to Mongolian Altai. Nevertheless, the finding is noteworthy as rather distant from the closest localities and being the first record of the species at the north-eastern macroslope of Mongolian Altai. Unlike other Mongolian localities representing the north-eastern limit of natural distribution area of the species, the new one ap- 
parently has anthropogenic character. It is unclear, however, whether this finding is occasional or $S$. brassiciforme has the potential of expansion of its distribution under the natural conditions of W Mongolia; in the latter case, further findings at NE slope of Mongolian Altai and neighboring regions can be assumed.

\section{Taxonomic remarks}

\section{Guenthera Andrz.}

Gómez-Campo (2003), using morphological and primary molecular evidences, argued for the restoration of the genus Guenthera Andrz. as distinct from Brassica L., and this viewpoint was supported by subsequent phylogenetic studies (Warwick, Hall, 2009; Arias, Pires, 2012). The weedy SW Asian species introduced in Mongolia and elsewhere is usually treated either as a subspecies of $B$. elongata Ehrh. [B. elongata subsp. integrifolia (Boiss.) Breistr.] or as a distinct species B. armoracioides Czern. ex Turcz. (Erucastrum armoracioides (Czern. ex Turcz.) Cruchet). The latter viewpoint is preferred by me and relevant combination based ont the prior basionym $B$. persica is given below. As long as other subspecies of G. elongata (Ehrh.) Andrz. recognized by Gómez-Campo reveal at least the same level of differences from subsp. elongata as subsp. integrifolia (Boiss.) Gómez-Campo does, they are also accepted here as specifically distinct from G. elongata.

Guenthera cretacea (Kotov) D. A. German, comb. nov. - Erucastrum cretaceum Kotov, 1939, Zhurn. Inst. Bot. Vseukraïns'k. Akad. Nauk 21-22: 237. - Brassica cretacea (Kotov) Stank. ex Tzvel., 2003, Novit. Syst. P1. Vasc. 35: 107. - B. elongata subsp. pinnatifida (Schmalh.) Greuter et Burdet, 1985, Willdenowia 15 (1): 64. - G. elongata subsp. pinnatifida (Schmalh.) Gómez-Campo, 2003, Anales Jard. Bot. Madrid 60: 304.

Guenthera persica (Boiss. et Hohen.) D. A. German, comb. nov. - Brassica persica Boiss. et Hohen., 1849, Diagn. Pl. Or. Nov. 8: 26.

Guenthera subscaposa (Maire et Weiller) D. A. German, comb. nov. - Brassica subscaposa Maire et Weiller, 1940, Bull. Soc. Hist. Nat. Afrique N. 31: 9. - B. elongata subsp. subscaposa (Maire et Weiller) Maire, 1965, Fl. Afrique N. 12: 168. G. elongata subsp. subscaposa (Maire et Weiller)
Gómez-Campo, 2003, Anales Jard. Bot. Madrid 60: 304.

Guenthera subscaposa subsp. imdrahsiana (Quézel) D. A. German, comb. nov. - Brassica elongata subsp. imdrahsiana Quézel, 1955, Bull. Soc. Sci. Nat. Maroc 34: 304. - G. elongata subsp. imdrahsiana (Quézel) Gómez-Campo, 2003, Anales Jard. Bot. Madrid 60: 304.

Rather recently described Erucastrum takhtajanii V. I. Dorof. (Dorofeyev, 2000) characterized by well-developed indumentum of stems, leaves, pedicels, and sepals, which I previously accepted as a subspecies of B. elongata (German, 2005c), appears to be similar to B. elongata var. scabra O. E. Schulz and could be treated as a variety of either G. persica or G. elongata subsp. integrifolia.

\section{Stevenia Fisch.}

Stevenia cheiranthoides subsp. incarnata (Kamelin) D. A. German, comb. et stat. nov. S. incarnata Kamelin, 1995, Bot. Zhurn. 80, 3: 73.

The first attempt to validate this combination (German, 2010: 84) turned to be unsuccessful on account of the invalidity of the intended basionym cited as "Arabis incarnata Pall. ex DC., Reg. Veg. Syst. Nat. 2: 210. 1821". As evidenced by the way this variety was cited in Candolle (1821, 1824, both times as " $\beta$ Arabis incarnata Pall.! in herb. Lamb."), the author did not intend to name it and just cited herbarium material annotated as "Arabis incarnata Pall.". Hence, neither the correction of the "basionym" to "Stevenia cheiranthoides var. ( $\beta$ ) incarnata Pall. ex DC." suggested by German (2011b: 25) is possible, nor the binominal $S$. incarnata published as if being based on "Arabis incarnata" (Kamelin, 1995) can be considered as such. However, direct reference to the previously and effectively published diagnosis of Candolle's "var. $\beta$ " in the protologue of $S$. incarnata along with the type designation made the last binominal a validly published name of a new taxon. In German (2011), a full and direct reference to the potential basionym, $S$. incarnata, was given but the combination "S. cheiranthoides subsp. incarnata" was not definitely accepted which precluded its validation (ICN Art. 36.1(a); McNeill et al., 2012) that is finally done here.

Acknowledgements. Curators and managers of the visited herbaria and their departments, namely 
Alexander I. Shmakov, Nadezhda A. Usik, Zoltán Barina, Klaus Pistrick, Matthias H. Hoffmann, Rudolf V. Kamelin, Alice E. Grabovskaya-Borodina, Irina D. Illarionova, Alexey P. Seregin, Sergey A. Balandin, Tatyana P. Balandina, Barbara Neuffer, Herbert Hurka, Nikolai W. Friesen, Otakar Š́́da, and Irina I. Gureyeva are thanked for providing the opportunity of working with collections. Kind sending the images of certain specimens and/or literature sources by Evgeny V. Boltenkov and Batlai Oyuntsetseg is highly appreciated. John McNeill, John Wiersema, Kanchi Gandhi, and Alexander N. Sennikov are much thanked for nomenclatural expertise of Stevenia cheiranthoides subsp. incarnata case. For valuable comments on the manuscript, I am grateful to Alexander L. Ebel.

\section{LITERATURE}

Ahti T. On the taxonomy of Erysimum cheiranthoides L. (Cruciferae) // Arch. Soc. Zool. Bot. Fenn. "Vanamo", 1961. - Vol. 16, No. 1. - P. 22-35.

Al-Shehbaz I.A. A revision of the Himalayan and Central Asian genus Taphrospermum (Brassicaceae) // Harvard Pap. Bot., 2000. - Vol. 5, No. 1. - P. 99-108.

Al-Shehbaz I.A. Nomenclatural notes on Eurasian Arabis (Brassicaceae) // Novon, 2005. - Vol. 15, No. 4. - P. 519-524.

Al-Shehbaz I.A. A generic and tribal synopsis of the Brassicaceae (Cruciferae) // Taxon, 2012. - Vol. 61, No. 5. - P. 931-954.

Al-Shehbaz I.A., German D.A. A synopsis of the genus Braya (Brassicaceae) // Harvard Papers in Botany, 2014. Vol. 19, No. 2. - P. 161-174. [doi: 10.3100/hpib.v19iss2.2014.n1]

Al-Shehbaz I.A., Ohba H. The status of Dimorphostemon and two new combinations in Dontostemon (Brassicaceae) // Novon, 2000. - Vol. 10, No. 1. - P. 95-98.

Al-Shehbaz I.A., Warwick S.I. A synopsis Eutrema (Brassicaceae) // Harvard Pap. Bot., 2005. - Vol. 10, No. 2. - P. 129-135.

Al-Shehbaz I.A., O'Kane S.L., Price R.A. Generic placement of species excluded from Arabidopsis (Brassicaceae) // Novon, 1999. - Vol. 9, No. 3. - P. 296-307.

Al-Shehbaz I.A., Mummenhoff K., Appel O. Cardaria, Coronopus, and Stroganowia are united with Lepidium (Brassicaceae) // Novon, 2002. - Vol. 12, No. 1. - P. 5-11.

Al-Shehbaz I.A., Windham M.D., Elven R. Draba Linnaeus // Flora of North America, 2010. - Vol. 7. - P. 269-347.

Al-Shehbaz I.A., German D.A., Moazzeni H., Mummenhoff K. Systematics, tribal placements, and synopses of the Malcolmia s. 1. segregates (Brassicaceae) // Harvard Papers in Botany, 2014. - Vol. 19, No. 1. - P. 53-71. [doi: 10.3100/hpib.v19iss1.2014.n4]

Appel O., Al-Shehbaz I.A. Generic limits and taxonomy of Hornungia, Pritzelago, and Hymenolobus (Brassicaceae) // Novon, 1997. - Vol. 7, No. 4. - P. 338-340.

Arias T., Pires J.Ch. A fully resolved chloroplast phylogeny of the brassica crops and wild relatives (Brassicaceae: Brassiceae): Novel clades and potential taxonomic implications // Taxon, 2012. - Vol. 61, No. 5. - P. 980-988.

Bobrov E.G. Species Georgianae neglectae e "Flora Baicalensi" // Not. Syst. Herb. Inst. Bot. Acad. Sci. URSS, 1960. - Vol. 20. - P. 3-22 [in Russian].

Boczantzeva V.V. The new genus Galitzkya V. Boczantzeva (Cruciferae) // Bot. Zhurn., 1979. - Vol. 64, No. 10. - P. 1440-1442 [in Russian].

Botschantzev V.P. Generis Goldbachiae DC. revisio // Not. Syst. Herb. Inst. Bot. Acad. Sci. URSS, 1963. - Vol. 22. - P. 135-143 [in Russian].

Botschantzev V.P. The genus Strigosella Boiss. and its relation to the genus Malcolmia R. Br. (Cruciferae) // Bot. Zhurn., 1972. - Vol. 57, No. 9. - P. 1033-1046 [in Russian].

Botschantzev V.P. Two new genera of the family Cruciferae // Bot. Zhurn., 1980. - Vol. 65, No. 3. - P. 425-427 [in Russian].

Buzunova I.O. Brassicaceae Burnett (Cruciferae Juss.) // Catalogue of the type specimens of Central Asian vascular plants in the Herbarium of the V. L. Komarov Botanical Institute / Ed. by V.I. Grubov. - St. Petersburg: St. Petersburg University Press, 2000. - P. 66-76 [in Russian].

Candolle A.P., de. Prodromus systematis naturalis regni vegetabilis. - P. 1. - Parisiis: Sumptibus Sociorum Treuttel et Würtz, 1824. - $748 \mathrm{p}$.

Candolle A.P., de. Regni vegetabilis systema naturale. - Vol. 2. - Parisiis: Socii Treuttel et Würtz, 1821. - [II] + $745 \mathrm{p}$.

Chernyakovskaya E.G. Matthiola R. Br. // Flora URSR / Ed. by V.L. Komarov. - Moscow \& Leningrad, 1939. Vol. 8. - P. 285-298 [in Russian]. 
Darijmaa Sh., Bayanmunkh T., Jimsee D. Flora of the nearest in Ikh Nart station // Proc. Inst. Bot., Mongolian Academy of Sciences, 2004. - Vol. 15. - P. 68-73 (in Mongolian).

Dorofeyev V.I. Genus Erucastrum (Brassicaceae) in Caucasian flora // Bot. Zhurn., 2000. - Vol. 85, No. 7. - P. 183-185 [in Russian].

Doronkin V., Shaulo D., Han I., Vlasova N., Ivleva V., Enkhtuya L., Munkh-Erdene T., Ochgerel N., Munkhjargal B. New records for the flora of Selenge Province (Mongolia) // Skvortsovia, 2015. - Vol. 2, No. 1. - P. 8-27.

Dulamsuren Ch., Mühlenberg M. Additions to the flora of the Khentei, Mongolia // Willdenowia, 2003. - Vol. 33, No. 1. - P. 149-158.

Ebel A.L. Genus Smelowskia (Brassicaceae) in South Siberia and Mongolia // Natural conditions, history, and culture of West Mongolia and adjacent regions / Proceedings of $4^{\text {th }}$ International Conference (Tomsk, April 20-24, 1999). - Tomsk, 1999. - P. 138-139 [in Russian].

Ebel A.L. Notes on taxonomy and geography of Brassicaceae of Altai flora // Turczaninowia, 2000a. - Vol. 3, No. 3. - P. 18-43 [in Russian].

Ebel A.L. On the distribution of species of the genus Rorippa Scop. (Brassicaceae) in Siberia // Krylovia, 2000b. - Vol. 2, No. 1. - P. 81-86 [in Russian].

Ebel A.L. About two species of the genus Lepidium L. (sect. Dileptium DC.) in the flora of Siberia // Studies of Young Botanists of Siberia / Proceedings of the Conference of Young Scientists (Novosibirsk, February 20-22, 2001). - Novosibirsk, 2001. - P. 26-31 [in Russian].

Ebel A.L. Rare species of the genus Draba L. in the flora of Russian and Mongolian Altai / Proceedings of the $1^{\text {st }}$ International Scientific-Practical Conference (Barnaul, November 26-28, 2002). - Barnaul, 2002. - P. 77-82 [in Russian].

Ebel A.L., Rudaya N.A. Notes on flora of West Mongolia // Turczaninowia, 2002. - Vol. 5, No. 1. - P. $32-42$ [in Russian].

German D.A. To the check-list of Mongolian Cruciferae // Turczaninowia, 2001. - Vol. 4, No. 3. - P. $42-46$ [in Russian].

German D.A. On the distribution of species of the genus Dontostemon Andrz. ex C. A. Mey. (Cruciferae) in West Mongolia // Natural conditions, history and culture of Western Mongolia and adjacent regions / Theses of reports of VI International Scientific Conference (Mongolia, Khovd, 2003, September, 18-22). - Tomsk, 2003. - P. $78-80$ [in Russian].

German D.A. Type specimens of Altai Cruciferae taxa described in 1829-1841 by C. A. Meyer, A. A. Bunge and C. F. Ledebour deposited in the Herbarium of the Komarov Botanical Institute (LE) // Novosti Sist. Vyssh. Rast. [Novit. Syst. Pl. Vasc.], 2005a. - Vol. 37. - P. 233-267 [in Russian].

German D.A. Erysimum mongolicum, a new species from Mongolia, and notes on E. vassilczenkoi and E. kotuchovii (Brassicaceae) // Willdenowia, 2005b. - Vol. 35, No. 2. - P. 305-313.

German D.A. Two new combinations in the Caucasian Cruciferae // Turczaninowia, 2005c. - Vol. 8, No. 2. - P. 20-21 [in Russian].

German D.A. About the endemic species Erysimum transiliense (Cruciferae) // Sist. Zametki Mater. Gerb. Krylova Tomsk. Gosud. Univ. [Animadv. Syst. Herb. Kryl. Univ. Tomsk.].

German D.A. Critical notes on the genus Dontostemon (Brassicaceae) // Novon, 2007. - Vol. 17, No. 2. - P. $172-175$.

German D.A. Six new synonyms in the Central Asian Cruciferae (Brassicaceae) // Nordic J. Bot., 2008. - Vol. 25, No. 1-2. - P. 38-40.

German D.A. New data on the species composition and distribution of Mongolian mustards (Cruciferae) // Bot. Zhurn., 2009. - Vol. 94, No. 8. - P. 1149-1158 [in Russian].

German D.A. A checklist and the system of the Cruciferae of Altai // Komarovia, 2010 (as 2008). - Vol. 6, No. 2. - P. $80-88$.

German D.A. Typification of selected names of Cruciferae taxa from Siberia and some neighboring regions // Turczaninowia, 2011a. - Vol. 14, No. 1. - P. 45-54.

German D.A. Taxonomical confusions in the Cruciferae of North and Central Asia. I. Alyssum fischerianum and Alyssum canescens // Turczaninowia, 2011b. - Vol. 14, No. 4. - P. 18-28 [in Russian].

German D.A. Some additions to the flora of Afghanistan, China, Kyrgyzstan, and Mongolia from the family Cruciferae // Ann. Naturhist. Mus. Wien, ser. B, 2011c. - Bd. 112. - S. 503-505.

German D.A. Erysimum kamelinii, a new species from the Middle Asia and further additions to Polatschek's revision of the genus Erysimum (Cruciferae), part $1 / /$ Ann. Naturhist. Mus. Wien, ser. B, 2012a. - Bd. 113. - S. $266-272$.

German D.A. Typification of some names in the Cruciferae, mostly from North Asia // Turczaninowia, 2012b. Vol. 15, No. 3. - P. 9-16.

German D.A. Taxonomical confusions in the Cruciferae of North and Central Asia. III. Hesperis flava and Hesperis rupestris // Turczaninowia, 2012c. - Vol. 15, No. 4. - P. 9-18 [in Russian]. 
German D.A. (2112) Proposal to reject the name Sisymbrium lineare (Cruciferae) // Taxon, 2012d. - Vol. 61, No. 6. - P. 1331-1332.

German D.A. (2148) Proposal to reject the name Hesperis rupestris (Cruciferae) // Taxon, 2013a. - Vol. 62, No. 3. - P. 633-634.

German D.A. (2182) Proposal to conserve the name Alyssum canescens (Ptilotrichum canescens, Stevenia canescens) (Cruciferae) with a conserved type // Taxon, 2013b. - Vol. 62, No. 4. - P. 836-837. [doi: http://dx.doi. org/10.12705/624.18]

German D.A., Al-Shehbaz I.A. Dendroarabis, a new Asian genus of Brassicaceae // Harvard Papers in Botany, 2008. - Vol. 13, No. 2. - P. 289-291. [doi: http://dx.doi.org/10.3100/1043-4534-13.2.289]

German D.A., Al-Shehbaz I.A. Nomenclatural novelties in miscellaneous Asian Brassicaceae (Cruciferae) // Nordic Journal of Botany, 2010. - Vol. 28, No. 6. - P. 646-651. [doi: 10.1111/j.1756-1051.2010.00983.x]

German D.A., Chen W.-L. Notes on the family Brassicaceae in China // Journal of Systematics and Evolution, 2009. - Vol. 47, No. 3. - P. 202-219. [doi: 10.1111/j.1759-6831.2009.00022.x]

German D.A., Ebel A.L. Generic placement of Arabidopsis rupicola (Cruciferae) // Turczaninowia, 2005. - Vol. 8, No. 3. - P. 5-12 [in Russian].

German D.A., Ebel A.L. Some interesting records of Cruciferae in Asia // Sist. Zametki Mater. Gerb. Krylova Tomsk. Gosud. Univ. [Animadv. Syst. Herb. Kryl. Univ. Tomsk.], 2009. - Vol. 101. - P. 5-11 [in Russian].

German D.A., Oyuntsetseg B. Cruciferae (Brassicaceae) of Mongolia updated: comprehensive state of studies on taxonomy and geography of Mongolian mustards // Problems of Botany of South Siberia and Mongolia / Proceedings of the $7^{\text {th }}$ International Scientific-Practical Conference (Barnaul, October 21-24, 2008). - Barnaul: “Artika”, 2008. - P. $42-47$.

German D.A., Neuffer B., Friesen N., Hurka H. Contribution to the knowledge of the flora of the Mongolian Altai II // Feddes Repert., 2003. - Bd. 114. Hf. 7-8. - S. 632-637. [doi: 10.1002/fedr.200311018]

Gómez-Campo C. The genus Guenthera Andr. in Bess. (Brassicaceae, Brassiceae) // Anales Jard. Bot. Madrid, 2003. - Vol. 60, No. 2. - P. 301-307.

Grubov V.I. Conspect of the flora of Mongolian People Republic / Ed. by E.M. Lavrenko // Acta Mong. Comm. Acad. Sci. URSS, 1955. - Vol. 67. - P. 1-308 [in Russian].

Grubov V.I. Introduction // Rasteniya Tsentralnoy Azii. 1. Introduction. Filicales. Bibliography [Plantae Asiae Centralis. Fasc. 1. Praefatio. Filicales. Bibliographia] / Ed. by V.I. Grubov. - Moscow \& Leningrad: Izd-vo Akademii Nauk SSSR, 1963. - P. 5-69 [in Russian].

Grubov V.I. Addenda et corrigenda ad "Conspectum florae Respublicae Popularis Mongoliae" // Novosti Sist. Vyssh. Rast. [Novit. Syst. Pl. Vasc.], 1972. - Vol. 9. - P. 270-298 [in Russian].

Grubov V.I. The sixth supplement to the list of flora of Mongolian People's Republic // Bot. Zhurn., 1976. - Vol. 61, No. 12. - P. 1751-1753 [in Russian].

Grubov V.I. The seventh supplement to the list of flora of Mongolian People's Republic // Bot. Zhurn., 1977. - Vol. 62, No. 9. - P. 1309-1311 [in Russian].

Grubov V.I. The eighth supplement to the list of flora of Mongolian People's Republic // Bot. Zhurn., 1979. - Vol. 64, No. 3. - P. 403-405 [in Russian].

Grubov V.I. Key to the vascular plants of Mongolia (with an atlas). - Leningrad: Nauka, 1982. - 443 p. [in Russian].

Grubov V.I., Yunatov A.A. Main peculiarities of the flora of Mongolian People's Republic in relation to its division// Bot. Zhurn., 1952. - Vol. 37, No. 1. - P. 45-64 [in Russian].

Grubov V.I., Gubanov I.A., Darijmaa Sh. Floristic discoveries of 1989 in Mongolia // Byull. Moskovsk. Obshch. Isp. Prir. [Bull. Soc. Nat. Mosc.], Otd. Biol., 1990. - Vol. 95, No. 6. - P. 70-76 [in Russian].

Gubanov I.A. Conspectus of flora of Outer Mongolia (vascular plants). - Moscow: Valang, 1996. - 136 p. [in Russian].

Gubanov I.A. Additions and corrections to the "Conspectus of flora of Outer Mongolia (vascular plants)" // Turczaninowia, 1999. - Vol. 2, No. 3. - P. 19-23 [in Russian].

Gubanov I.A., Hilbig $\boldsymbol{W}$. Entwicklung und Stand der Gefusspflanzenflora des Mongolischen Volksrepublik // Erforsch. Biol. Ress. Mongolei (Halle/Saale), 1989. - Bd. 7. - S. 127-146.

Gubanov I.A., Kamelin R.V. New species of vascular plants revealed in the flora of Mongolia // Byull. Moskovsk. Obshch. Isp. Prir. [Bull. Soc. Nat. Mosc.], Otd. Biol., 1991. - Vol. 96, No. 6. - P. 112-115 [in Russian].

Gubanov I.A., Kamelin R.V., Budantsev A.L., Ganbold E., Darijmaa Sh. Plant species and genera new for Mongolia and some of its regions // Byull. Moskovsk. Obshch. Isp. Prir. [Bull. Soc. Nat. Mosc.], Otd. Biol., 1990. - Vol. 95, No. 1. - P. 117-123 [in Russian].

Gubanov I.A., Kamelin R.V., Ganbold E., Darijmaa Sh. Flora and vegetation of Khingan Mts. foothills and Khalkhin-gol river valley (Outer Mongolia) and their peculiarities // Byull. Moskovsk. Obshch. Isp. Prir. [Bull. Soc. Nat. Mosc.], Otd. Biol., 1996. - Vol. 101, No. 2. - P. 49-66 [in Russian]. 
Hanelt P., Davažamc S. [C.] Beitrag zur Kenntnis der Flora der Mongolische Volksrepublik, inbesondere des Gobi-Altai-, des Transaltai- und Alašan-Gobi-Bezirks // Feddes Repert., 1965. - Bd. 70. Hf. 1-3. - S. 7-68.

Hayasaka E. Vascular plants of Darhadyn Wetlands, Khuvsgul, Mongolia, collected in August 2005 and August 2006 // Bull. Fukui Bot. Gard., 2011. - Vol. 7. - P. 9-18.

Hilbig W., Schamsran Z. Beitrag zur Kenntnis der Flora des westlichen Teiles der Mongolischen Volksrepublik, speziell des Chovd Aimaks // Arch. Naturschutz. u. Landschaftsforsch., 1976. - Bd. 16. Hf. 2. - S. 141-162.

Hilbig W., Schamsran Z. Zweiter Beitrag zur Kenntnis der Flora des westlichen Teiles der Mongolischen Volksrepublik // Feddes Repert., 1980. - Bd. 91. Hf. 1-2. - S. 25-44.

Hilbig W., Schamsran Z. Dritter Beitrag zur Kenntnis der Flora der Mongolischen Volksrepublik // Feddes Repert., 1985. - Bd. 96. Hf. 4. - S. 285-298.

Hilbig W., Tungalag $\boldsymbol{R}$. Vegetationskundliche Untersuchungen in der Borzongijnund Galbyn-Gobi (Ömnögov Aimak, Mongolei) // Feddes Repert., 2006. - Bd. 117. Hf. 5-6. - S. 399-429.

Hilbig W., Pistrick K., Narantujaa N., Sančir Č. Beitrag zur Kenntnis der Flora der östlichen Mongolei (Ostmongolischer Florenbezirk und angrenzende Gebiete) // Erforsch. Biol. Ress. Mongolei (Halle/Saale), 2012. - Bd. 12. - S. 371-393.

Illarionova I. D. Synopsis of the genus Pugionium Gaertn. (Brassicaceae) // Komarovia, 1999. - Vol. 1. - P. $35-37$.

Jacquemoud F. Monographie du genre Sterigmostemum M. Bieb. (Cruciferae - Hesperideae) // Boissiera, 1988. - Vol. 40. - P. 8-161.

Jafri S.M.H. Brassicaceae // Ed. by E. Nasir, S.I. Ali. Flora of West Pakistan. - Vol. 55. - Karachi: Ferozsons, 1973. - P. 1-308.

Jäger E.J., Hanelt P., Davažamc C. Zur Flora der Dsungarischen Gobi (Mongolische Volksrepublik) // Flora, 1985. - Bd. 177. Hf. 1-2. - S. 45-89.

Kamelin R.V. Notes on Cruciferae of Siberia and Mongolia. Genus Stevenia // Bot. Zhurn., 1995. - Vol. 80, No. 3. - P. 65-78 [in Russian].

Kamelin R.V., German D.A. New species of the genus Sterigmostemum Bieb. (Cruciferae) from East Kazakhstan // Turczaninowia, 2001. - Vol. 4, No. 3. - P. 5-9 [in Russian].

Kamelin R.V., Gubanov I.A., Darijmaa Sh., Ganbold E. Floristic novelties from Outer Mongolia // Byull. Moskovsk. Obshch. Isp. Prir. [Bull. Soc. Nat. Mosc.], Otd. Biol., 1992. - Vol. 97, No. 5. - P. 60-71 [in Russian].

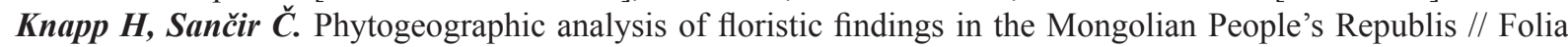
Geobot. Phytotax., 1986. - Vol. 12. - P. 287-324.

Knjasev M. S. Notes on some species of Brassicaceae in Urals and adjacent territories // Novosti Sist. Vyssh. Rast. [Novit. Syst. Pl. Vasc.], 2010. - Vol. 42. - P. 136-146 [in Russian].

Krasnoborov I.M., German D.A. Brassicaceae (Cruciferae) // Key for plants of Tuva Republic / Ed. by D.N. Shaulo. - Novosibirsk, Publishers of RAS, Siberian Branch, 2007. - P. 178-211 [in Russian].

Krylov P.N. Flora Sibiriae occidentalis. - Vol. 6. Papaveraceae - Saxifragaceae. - Tomsk, 1931. - P. 1229-1448 + I-XII.

Manibazar N. Dictionary of Latin and Mongolian names of vascular plants in Mongolia. - Ulaanbaatar, 2010. 244 p. (in Mongolian).

Maximowicz K.J. Diagnoses plantarum novarum asiaticarum // Bull. Acad. Sci. Pétersb., 1880. - Vol. 26. - P. 420-542.

Maximowicz K.J. Enumeratio plantarum hucusque in Mongolia nec non adjacenta parte Turkestaniae sinensis lectarum. - Petropoli, 1889. - Fasc. 1. - 146 p.

Meyer F.K. Conspectus der "Thlaspi"-arten Europas, Afrikas und Vorderasiens // Feddes Repert., 1973. - Bd. 84. Hf. 5-6. - S. 449-470.

Meyer F.K. Kritische Revision der “Thlaspi”-arten Europas, Vorderasiens und Afrikas I. Thlaspi L. // Haussknechtia, 2001. - Beih. 8. - S. 3-42.

Meyer F.K. Anmerkungen zu einigen Noccaea-Arten Nordasiens // Haussknechtia, 2010. - Beih. 12. - S. 5-18.

Neuffer B., Oyuntsetseg B., Schamsran Z., Friesen N., Hurka H. Contribution to the knowledge of the flora of the Mongolian Altai // Feddes Repert., 2003. - Bd. 114. Hf. 5-6. - S. 358-371.

Neuffer B., Friesen N., Oyuntsetseg B., Jamsran Ts., Hurka H. Osnabrück botanical expeditions to Mongolia // Erforsch. Biol. Ress. Mongolei (Halle/Saale), 2012. - Bd. 12. - S. 307-333.

Olonova M.V., Zhang D.-Y., Beket U. On the identification of important plant areas on Altai mountain country // Tomsk State Univ. J. Biol., 2013. - Vol. 1 (21). - P. 59-73 [in Russian].

Oyuntsetseg B. Brassicaceae Burnett, 1835 // Urgamal M., Oyuntsetseg B., Nyambayar D., Dulamsuren Ch. Conspectus of the vascular plants of Mongolia / Ed. by Ch. Sanchir, Ts. Jamsran. - Ulaanbaatar: "Admon" Press, 2014. - P. 158-169.

Polatschek A. Revision der Gattung Erysimum (Cruciferae): Teil 1: Russland, die Nachfolgestaaten der USSR (excl. Georgien, Armenien, Azerbaidzan), China, Indien, Pakistan, Japan und Korea // Ann. Naturhist. Mus. Wien, ser. B, 2010. - Bd. 111. - S. 181-275. 
Polozhij A.V., Balashova A.F. Types of taxa in P.N. Krylov Herbarium. - Tomsk: Publishers of Tomsk State University, 1989. - 48 p. [in Russian].

Revushkin A.S., Rudaya N.A., Smorgov A.E., Ebel A.L., Shchegoleva N.V. Floristic findings in West Mongolia // Bot. Zhurn., 2001. - Vol. 86, No. 5. - P. 142-151 [in Russian].

Rilke S., Najmi U. FloraGREIF - Virtual guide and plant data base as a practical approach to the flora of Mongolia // Willdenowia, 2011. - Vol. 41. No. 2. - P. 371-379.

Rilke S., Najmi U., Schnittler M. Contributions to "E-Taxonomy" - A virtual approach to the flora of Mongolia // Feddes Repert., 2012. - Bd. 123. Hf. 3. - S. 219-232. [doi: 10.1002/fedr.201200013]

Smirnov S.V., German D.A., Kossaczev P.A., Dyachenko S.A. Addition to the flora of Mongolia // Turczaninowia, 2003. - Vol. 6, No. 4. - P. 11-21 [in Russian].

Thellung A. Die Gattung Lepidium (L.) R. Br. Eine monographische Studie // Mitt. Bot. Univ. Zürich, 1906. - Bd. 27. - S. 1-340.

University of Greifswald, Institute of Botany and Landscape Ecology, Institute of Geography and Geology, Computer Centre, 2010 (continuously updated). FloraGREIF - Virtual Flora of Mongolia (http://greif.uni-greifswald.de/ floragreif/). Computer Centre of University of Greifswald, D-17487 Greifswald, Germany. [accessed 23 February 2015].

Urgamal M., Oyuntsetseg B., Nyambayar D. Synopsis and recent additions to the flora of Mongolia // Proc. Inst. Bot., Mongolian Academy of Sciences, 2013. - Vol. 25. - P. 53-72.

Urgamal M., Oyuntsetseg B., Nyambayar D., Dulamsuren Ch. Conspectus of the vascular plants of Mongolia / Ed. by Ch. Sanchir, Ts. Jamsran. - Ulaanbaatar: “Admon” Press, 2014. - 282 p.

Walters S.M., Akeroyd J.R. Draba L. // Flora Europaea / Ed. by T.G. Tutin, N.A. Burges, A.O. Chater, J.R. Edmondson, V.H. Heywood, D.M. Moore, D.H. Valentine, S.M. Walters, D.A. Webb. Vol. 1, ed. 2. - Cambridge, 1993. - P. 372-377.

Warwick S.I. \& Hall J.C. Phylogeny of Brassica and wild relatives (tribe Brassiceae: Brassicaceae) // Biology and breeding of crucifers / Ed. by S.K. Gupta. - London \& New York: Boca Raton: CRC Press, Taylor and Francis group, 2009. - P. 19-36.

Yang H.L. Cruciferae // Flora in desertis Reipublicae Populorum Sinarum. - Vol. 2. - Beijing: Science Press, 1987. P. 12-85 (in Chinese).

Yu Q.-Sh., Wang Q., Wanh A.-L., Wu G.-L., Liu J.-Q. Interspecific delimitation and phylogenetic origin of Pugionium (Brassicaceae) // Journal of Systematics and Evolution, 2010. - Vol. 48, No. 3. - P. 195-206.

Yu Q.-Sh., Wang Q., Wu G.-L., Ma Y.Zh., He X.Y., Wang X., Xie P.H., Hu L.H., Liu J.-Q. Genetic differentiation and delimitation of Pugionium dolabratum and Pugionium cornutum (Brassicaceae) // Plant Systematics and Evolution, 2013. - Vol. 299, No. 7. - P. 1355-1365. [doi: 10.1007/s00606-013-0800-3]

Zemmrich A., Schnittler M., Hartleib J., Busch M., Bobertz, B., Zölitz, R. FloraGREIF - an internet-based data repository for biogeographical research in Mongolia // Folia Geobot., 2013. - Vol. 48. - P. 523-536. [doi: 10.1007/ s12224-012-9149-6]

Zhou T.Y., Lu L.L., Yang G., Al-Shehbaz I.A. Brassicaceae (Cruciferae) // Flora of China / Ed. by Zh.G. Wu, P.H. Raven. - Vol. 8 (Brassicaceae through Saxifragaceae). - Beijing: Science Press and St. Louis: Missouri Botanical Garden Press, 2001. - P. 1-193. 